MATHEMATICS OF COMPUTATION

Volume 77, Number 261, January 2008, Pages 93-123

S 0025-5718(07)01912-6

Article electronically published on June 18, 2007

\title{
CONVERGENCE OF CLASSES OF HIGH-ORDER SEMI-LAGRANGIAN SCHEMES FOR THE VLASOV-POISSON SYSTEM
}

\author{
NICOLAS BESSE AND MICHEL MEHRENBERGER
}

\begin{abstract}
In this paper we present some classes of high-order semi-Lagrangian schemes for solving the periodic one-dimensional Vlasov-Poisson system in phase-space on uniform grids. We prove that the distribution function $f(t, x, v)$ and the electric field $E(t, x)$ converge in the $L^{2}$ norm with a rate of

$$
\mathcal{O}\left(\Delta t^{2}+h^{m+1}+\frac{h^{m+1}}{\Delta t}\right)
$$

where $m$ is the degree of the polynomial reconstruction, and $\Delta t$ and $h$ are respectively the time and the phase-space discretization parameters.
\end{abstract}

\section{INTRODUCTION}

While satisfactory numerical results have been presented in many papers on fluid mechanics and plasma physics (cf. 22, 8, 13, 25]) using semi-Lagrangian methods, only a few rigorous convergence analyses of these methods can be found. In spite of interesting a priori estimates pointed out in [3, 4, 14] for some particular cases, more general situations still remain to be rigorously worked out for convergence. The most difficult step in this convergence analysis is the determination of a stability result for the interpolation operators. While the stability results in $L^{\infty}$ norm seems inaccessible for high-order interpolation operators because of the Runge phenomenon (the artificial oscillations, whose amplitudes increase with the degree of the polynomials in the case of Lagrange interpolation, appear at the edges of the finite elements), a more appropriate mathematical framework is the $L^{2}$ stability. In this paper we continue the numerical analysis of semi-Lagrangian methods started in [6, 7]. The high-order error estimates announced in the paper [6] are rigorously proved in the present one. In the reference 8 ] we introduced new semi-Lagrangian schemes for unstructured meshes of phase space in order to deal with complex geometry and to use the local mesh refinement. These schemes use finite elements type reconstructions such as the Lagrange and the Hermite type interpolations, for which the gradients should be also transported (see [8]). The Hermite type interpolation leads to high-order, stable and slightly diffusive schemes, whereas the Lagrange interpolation leads to convergent but too diffusive schemes for orders smaller than two and unstable schemes in any $L^{p}$ norm for orders greater than two. Nevertheless, on uniform grids, if we use symmetric Lagrange interpolation we can then recover the stability in the $L^{2}$ discrete norm and keep the high-order

Received by the editor March 29, 2005 and, in revised form, May 25, 2005.

2000 Mathematics Subject Classification. Primary 65M12.

(C)2007 American Mathematical Society Reverts to public domain 28 years from publication 
and weak diffusion features of our approximation. In the present paper we give the convergence proof of such schemes. The case of uniform grids is interesting not only because the analysis is more simple but also because it gives satisfactory results in many physical applications [2, 24. In the paper [6] a convergence proof of a scheme defined on a triangulation using first-order Lagrange interpolation was given in the $L^{\infty}$ framework. The convergence proof and the error estimates for a semi-Lagrangian scheme with propagation of gradients on uniform grids (which use Hermite type interpolation) were given in 7 . If the main ideas of the present proof are contained in [6], the $L^{2}$ discrete framework implies different estimates and obliges us to manipulate carefully the interpolation operators in a tricky way which is very different from the one that has been carried out in [6]. Moreover the key of the proof also resides in the precise and fine study of the stability of the interpolation operator. This study is generally obvious for lower-order interpolation, but it becomes evidently difficult for high-order interpolation. In [12] the convergence for an adaptive semi-Lagrangian scheme using first-order Lagrange interpolation has been proved. But we cannot expect to obtain high-order estimates with this latter algorithm because we are restricted by the stability region phenomenon. Nevertheless, using the interpolatory wavelets of Deslaurier and Dubuc (wavelet built on Lagrange interpolation of any order) we could succeed in showing the convergence of the high-order adaptive schemes (see 9 ).

This paper is organized as follows. In the first part we present the continuous problem. Then the second part deals with the discrete problem and the numerical scheme to solve it. We study the convergence of our numerical scheme in a third part. In the Appendix, we give new proofs of some interpolation properties and recall some other proofs which are crucial for the $L^{2}$ stability.

\section{The COntinuous PRoblem}

2.1. The Vlasov-Poisson model. Denoting by $f(t, x, v) \geq 0$ the distribution function of electrons in phase-space (with the mass and the charge normalized to one), and by $E(t, x)$ the self-consistent electric field, the adimensional VlasovPoisson system reads

$$
\begin{gathered}
\frac{\partial f}{\partial t}+v \frac{\partial f}{\partial x}+E(t, x) \frac{\partial f}{\partial v}=0 \\
\frac{d E}{d x}(t, x)=\rho(t, x)=\int_{-\infty}^{+\infty} f(t, x, v) d v-1,
\end{gathered}
$$

where $x$ and $v$ are independent variables. We consider a plasma of period $L$. Hence, in (11) and (2) we have $x \in[0, L], v \in \mathbb{R}, t \geq 0$, and the functions $f$ and $E$ satisfy the periodic boundary conditions

$$
f(t, 0, v)=f(t, L, v), \quad v \in \mathbb{R}, \quad t \geq 0,
$$

and

$$
E(t, 0)=E(t, L) \Longleftrightarrow \frac{1}{L} \int_{0}^{L} \int_{-\infty}^{+\infty} f(t, x, v) d v d x=1, t \geq 0,
$$

which means that the plasma is globally neutral. In order to have a well-posed problem, we add to equations (11) -(4) a zero-mean electrostatic condition:

$$
\int_{0}^{L} E(t, x) d x=0, \quad t \geq 0
$$


and a initial condition

$$
f(0, x, v)=f_{0}(x, v), \quad x \in[0, L], \quad v \in \mathbb{R} .
$$

Assuming a smooth-enough electric field $E$ we can solve the equations (11), (3) and (6) in the classical sense as follows. We refer the reader to [10] for the existence, the uniqueness, and the regularity of the solutions of the following differential system.

We now consider the first-order differential system

$$
\begin{aligned}
& \frac{d X}{d t}(t ; s, x, v)=V(t ; s, x, v), \\
& \frac{d V}{d t}(t ; s, x, v)=E(t, X(t ; s, x, v)) .
\end{aligned}
$$

We denote it by the characteristic curves $t \rightarrow(X(t ; s, x, v), V(t ; s, x, v))$ which are the solutions of (7) subjected to the initial condition

$$
X(s ; s, x, v)=x, \quad V(s ; s, x, v)=v .
$$

The solution of problems (11) and (6) is then given by

$$
f(t, x, v)=f_{0}(X(0 ; t, x, v), V(0 ; t, x, v)), \quad x, v \in \mathbb{R}, \quad t \geq 0 .
$$

We note that the periodicity in $x$ of $f_{0}(x, v)$ and $E(t, x)$ implies the periodicity in $x$ of $f(t, x, v)$. Moreover, as

$$
\left|\frac{\partial(X, V)}{\partial(x, v)}\right|=1
$$

we get

$$
\frac{1}{L} \int_{0}^{L} \int_{-\infty}^{+\infty} f(t, x, v) d v d x=\frac{1}{L} \int_{0}^{L} \int_{-\infty}^{+\infty} f_{0}(x, v) d v d x=1
$$

Therefore, according to the previous considerations, the Vlasov-Poisson periodic problem consists in finding a couple $(f, E)$, smooth enough, periodic with respect to $x$, with period $L$, which solves the equations (2), (7), (8), and (9). Introducing the electrostatic potential $\phi=\phi(t, x)$ such that $E(t, x)=-\partial_{x} \phi(t, x)$, and denoting by $G=G(x, y)$ the fundamental solution of the Laplacian operator in one dimension (i.e. $-\partial_{x}^{2} G(x, y)=\delta(x-y)$ ) with periodic boundary condition, we obtain

$$
E(t, x)=\int_{0}^{L} K(x, y)\left(\int_{-\infty}^{+\infty} f(t, y, v) d v-1\right) d y
$$

where

$$
K(x, y)=-\partial_{x} G(x, y)= \begin{cases}\left(\frac{y}{L}-1\right) & 0 \leq x<y \\ \frac{y}{L} & y<x \leq L .\end{cases}
$$

2.2. Existence, uniqueness, and regularity of the solution of the continuous problem. In this section we recall the theorem of the existence of classical solutions for the Vlasov-Poisson system. The following theorem gives the existence, the uniqueness, and the regularity of the time-global classical solution of the Vlasov-Poisson periodic system in one dimension. Let $W^{1, \infty}$ be the Sobolev space consisting of all functions $\phi$ which, together with all partial derivatives $D^{\alpha} \phi$ taken in the sense of distributions of order $|\alpha| \leq 1$, belong to $L^{\infty}$ space. We then define $W_{c, \text { per }}^{1, \infty}\left(\mathbb{R}_{x} \times \mathbb{R}_{v}\right)$ as the subspace of $W^{1, \infty}\left(\mathbb{R}_{x} \times \mathbb{R}_{v}\right)$ consisting of those functions $\phi$ for which $0 \leq|\alpha| \leq 1$ and $D^{\alpha} \phi$ is periodic in $x$ and has compact support in $v$. 
Theorem 1. Assume that $f_{0} \in W_{c, p e r_{x}}^{1, \infty}\left(\mathbb{R}_{x} \times \mathbb{R}_{v}\right)$ is positive, periodic in $x$ with period $L$, and $Q(0) \leq R$ with $R>0$, and that $Q(t)$ is defined as follows:

$$
Q(t)=1+\sup \{|v|: \exists x \in[0, L], \quad \tau \in[0, t] \mid f(\tau, x, v) \neq 0\},
$$

and

$$
\frac{1}{L} \int_{0}^{L} \int_{-\infty}^{+\infty} f_{0}(x, v) d v d x=1
$$

Then the periodic Vlasov-Poisson system has a unique classical solution $(f, E)$ that is periodic in $x$, with period $L$, for all time $t$ in $[0, T]$, such that

$$
\begin{gathered}
f \in W^{1, \infty}\left(0, T ; W_{c, \text { per }_{x}}^{1, \infty}\left(\mathbb{R}_{x} \times \mathbb{R}_{v}\right)\right), \\
E \in W^{1, \infty}\left(0, T ; W_{\text {per }_{x}}^{1, \infty}(\mathbb{R})\right),
\end{gathered}
$$

and there exists a constant $C=C\left(R, f_{0}\right)$ dependent of $R$ and $f_{0}$ such that

$$
Q(T) \leq C T \text {. }
$$

Moreover, if we assume that $f_{0} \in W_{c, \text { per }}^{m, \infty}\left(\mathbb{R}_{x} \times \mathbb{R}_{v}\right)$, then

$$
(f, E) \in W^{m, \infty}\left(0, T ; W_{c, p e r_{x}}^{m, \infty}\left(\mathbb{R}_{x} \times \mathbb{R}_{v}\right)\right) \times W^{m, \infty}\left(0, T ; W_{\text {per }_{x}}^{m, \infty}(\mathbb{R})\right),
$$

for all finite time $T$.

Proof. We refer the reader to the review papers [18] or [10] for the proof.

\section{The Discrete PROBLEM}

3.1. Definitions and notation. Let $\Omega=[0, L] \times[-R, R]$, with $R>Q(T)$, and let $\mathcal{M}_{h}$ be a cartesian mesh of the phase-space $\Omega$. The mesh $\mathcal{M}_{h}$ is then given by two increasing sequences $\left(x_{i}\right)_{i \in\left\{0, \ldots, N_{x}\right\}}$ and $\left(v_{i}\right)_{i \in\left\{0, \ldots, N_{v}\right\}}$, respectively, of the intervals $[0, L]$ and $[-R, R]$. Let $\Delta x_{i}=x_{i+1}-x_{i}$ be the physical space set and $\Delta v_{i}=v_{i+1}-v_{i}$ the velocity space set. In order to simplify the convergence analysis we suppose that $\Delta x_{i}=\Delta x=L /\left(N_{x}+1\right)$ and $\Delta v_{i}=\Delta v=2 R /\left(N_{v}+1\right)$, where $N_{x}, N_{v} \in \mathbb{N}$. We can then define $h=\max \{\Delta x, \Delta v\}$.

For each function $g$ defined on all the points $\left(x_{i}, v_{j}\right) \in \mathcal{M}_{h}$, we shall write $g_{i, j}:=g\left(x_{i}, v_{j}\right)$ and complete the sequence on $\mathbb{Z} \times \mathbb{Z}$ by periodicity, by setting $g_{i, j}:=g_{i \bmod N_{x}+1, j \bmod N_{v}+1}$. The sequence $\left(x_{i}, v_{j}\right)$ will also be defined on the whole set $\mathbb{Z} \times \mathbb{Z}$ by $x_{i}:=i \Delta x$ and $v_{j}:=-R+j \Delta v$. We denote by $\mathcal{P}(\Omega)$ the set of all $\Omega$-periodic functions ( $L$-periodic in $x$ and $2 R$-periodic in $v$ ). We have $g_{i, j}=g\left(x_{i}, v_{j}\right)$ for $g \in \mathcal{P}(\Omega)$ and for all indices $(i, j) \in \mathbb{Z} \times \mathbb{Z}$. In fact, we will see that all functions which we consider on $\mathbb{R}_{x} \times \mathbb{R}_{v}$ belong to $\mathcal{P}(\Omega)$. Now let $f=\left\{f_{i, j}\right\}_{(i, j) \in \mathbb{Z} \times \mathbb{Z}}$ be a periodic grid-function with, respectively, the period $N_{x}+1$ and $N_{v}+1$. If $f$ is a function defined on the points of $\mathcal{M}_{h}$, we can associate to it a grid-function $\tilde{f}$ defined by $\tilde{f}_{i, j}:=f\left(x_{i}, v_{j}\right)$ for all $(i, j)=\mathbf{0}, \ldots, \mathbf{N}$ setting $\mathbf{N}=\left(N_{x}, N_{v}\right)$ and $\mathbf{0}=(0,0)$. We will replace $\tilde{f}$ by $f$ in order to simplify the notation. Let $L_{h}^{2}(\Omega)$ be the set of grid-functions whose norm $\|\cdot\|_{L_{h}^{2}(\Omega)}$ is bounded with

$$
\|f\|_{L_{h}^{2}(\Omega)}=\left(\Delta x \Delta v \sum_{(i, j)=\mathbf{0}}^{\mathbf{N}}\left|f_{i, j}\right|^{2}\right)^{1 / 2}
$$


As in the continuous case, we can define a discrete scalar product $\langle., .\rangle_{L_{h}^{2}(\Omega)}$ as follows. Let $f$ and $g$ be two complex-valued grid-functions of $L_{h}^{2}(\Omega)$. The scalar product $\langle f, g\rangle_{L_{h}^{2}(\Omega)}$ is then defined as

$$
\langle f, g\rangle_{L_{h}^{2}(\Omega)}=\Delta x \Delta v \sum_{(i, j)=\mathbf{0}}^{\mathbf{N}} f_{i, j} \overline{g_{i, j}} .
$$

Let $\boldsymbol{\omega}=\left(\omega_{x}, \omega_{v}\right)$ be a two-components vector of $\mathbb{Z}^{2}$. Let $\mathbf{z}$ and $\mathbf{k}$ be abbreviations, respectively, for $(x, v)$ and $\left(k_{x}, k_{v}\right)$. Therefore, $k_{x}$ (resp. $k_{v}$ ) takes the values $2 \pi \omega_{x} / L$ (resp. $2 \pi \omega_{v} /(2 R)$ ), where $\omega_{x}$ (resp. $\omega_{v}$ ) is an integer and the indices $(i, j) \in \mathbb{Z}^{2}$ correspond to the point $\mathbf{z}_{i, j}:=\left(x_{i}, v_{j}\right)$ in the phase space. If we now define

$$
\phi_{\boldsymbol{\omega}}(x, v)=\phi_{\boldsymbol{\omega}}(\mathbf{z})=\frac{e^{i\langle\mathbf{k}(\boldsymbol{\omega}), \mathbf{z}\rangle}}{|\Omega|^{1 / 2}}
$$

with $\langle\mathbf{k}(\boldsymbol{\omega}), \mathbf{z}\rangle=\frac{2 \pi \omega_{x}}{L} x+\frac{2 \pi \omega_{v}}{2 R} v$, the functions $\left\{\phi_{\boldsymbol{\omega}}\right\}_{\boldsymbol{\omega}=\mathbf{0}}^{\mathbf{N}}$ therefore form an orthonormal system on the grid $\mathcal{M}_{h}$ for the scalar product $\langle., .\rangle_{L_{h}^{2}(\Omega)}$. We then have

$$
\left\langle\phi_{\boldsymbol{\omega}}, \phi_{\boldsymbol{\nu}}\right\rangle_{L_{h}^{2}(\Omega)}=\delta_{\boldsymbol{\omega}, \boldsymbol{\nu}}
$$

If the function $\phi=\sum_{\boldsymbol{\omega}=\mathbf{0}}^{\mathbf{N}} \widehat{f}(\boldsymbol{\omega}) \phi_{\boldsymbol{\omega}}$ interpolates $f$ at the $\mathcal{M}_{h}$-grid points, then we have $f=\phi$ on $\mathcal{M}_{h}$ and

$$
\left\langle\phi, \phi_{\boldsymbol{\omega}}\right\rangle_{L_{h}^{2}(\Omega)}=\left\langle f, \phi_{\boldsymbol{\omega}}\right\rangle_{L_{h}^{2}(\Omega)}=\sum_{\boldsymbol{\nu}=\mathbf{0}}^{\mathbf{N}} \widehat{f}(\boldsymbol{\nu})\left\langle\phi_{\boldsymbol{\nu}}, \phi_{\boldsymbol{\omega}}\right\rangle_{L_{h}^{2}(\Omega)}=\widehat{f}(\boldsymbol{\omega}),
$$

where

$$
\widehat{f}(\boldsymbol{\omega})=\frac{1}{|\Omega|^{1 / 2}} \sum_{(i, j)=\mathbf{0}}^{\mathbf{N}} f_{i, j} e^{-i\left\langle\mathbf{k}(\boldsymbol{\omega}), \mathbf{z}_{i, j}\right\rangle}, \quad \boldsymbol{\omega}=(0,0),(1,0),(0,1), \ldots, \mathbf{N} .
$$

Obviously if $\phi=\sum_{\boldsymbol{\omega}=\mathbf{0}}^{\mathbf{N}} \widehat{f}(\boldsymbol{\omega}) \phi_{\boldsymbol{\omega}}$ interpolates $f$ at the $\mathcal{M}_{h}$-grid points, then

$$
f_{i, j}=\frac{1}{|\Omega|^{1 / 2}} \sum_{\boldsymbol{\omega}=\mathbf{0}}^{\mathbf{N}} \widehat{f}(\boldsymbol{\omega}) e^{i\left\langle\mathbf{k}(\boldsymbol{\omega}), \mathbf{z}_{i, j}\right\rangle}, \quad i=0, \ldots, N_{x}, \quad j=0, \ldots, N_{v} .
$$

Moreover, we also have a discrete version of the Parseval equality

$$
\|f\|_{L_{h}^{2}(\Omega)}^{2}=\Delta x \Delta v \sum_{(i, j)=\mathbf{0}}^{\mathbf{N}}\left|f_{i, j}\right|^{2}=\sum_{\boldsymbol{\omega}=\mathbf{0}}^{\mathbf{N}}|\widehat{f}(\boldsymbol{\omega})|^{2} .
$$

The formulaes (10) and (11) are the direct and inverse discrete Fourier transforms. We set $\eta_{x}=N_{x} / 2$ and $\theta_{x}=0$ if $N_{x}$ is even, and $\eta_{x}=\left(N_{x}-1\right) / 2$ and $\theta_{x}=1$ if $N_{x}$ is odd. Similarly, we set $\eta_{v}=N_{v} / 2$ and $\theta_{v}=0$ if $N_{v}$ is even, and $\eta_{v}=\left(N_{v}-1\right) / 2$ and $\theta_{v}=1$ if $N_{v}$ is odd. Instead of varying $\omega_{x}\left(\right.$ resp. $\left.\omega_{v}\right)$ from 0 to $N_{x}\left(\right.$ resp. $\left.N_{v}\right)$, $\omega_{x}\left(\right.$ resp. $\left.\omega_{v}\right)$ is varied from $-\eta_{x}$ (resp. $-\eta_{v}$ ) to $\eta_{x}+\theta_{x}$ (resp. $\eta_{v}+\theta_{v}$ ). To simplify the notation, without loss of generality, $N_{x}$ and $N_{v}$ are taken even. We use the notation

$$
\sum_{|\omega| \leq \mathbf{N} / 2}=\sum_{\left|\omega_{x}\right| \leq N_{x} / 2} \sum_{\left|\omega_{v}\right| \leq N_{v} / 2}=\sum_{\omega_{x}=-N_{x} / 2}^{N_{x} / 2} \sum_{\omega_{v}=-N_{v} / 2}^{N_{v} / 2} .
$$


Let $\boldsymbol{\alpha}$ and $\boldsymbol{\beta}$ be, respectively, the real vectors $\left(\alpha_{0}, \ldots, \alpha_{j}, \ldots, \alpha_{N_{v}}\right)$ and $\left(\beta_{0}, \ldots, \beta_{i}\right.$, $\left.\ldots, \beta_{N_{x}}\right)$ with $0 \leq \alpha_{j}, \beta_{i} \leq 1, \forall(i, j) \in\left[0, N_{x}\right] \times\left[0, N_{v}\right]$. We can therefore define the norm $\|\cdot\|_{L_{h}^{2}, \Delta_{h}^{\alpha, \beta}}$ by

$$
\|f\|_{L_{h}^{2}, \Delta_{h}^{\alpha, \beta}}=\left(\Delta x \Delta v \sum_{(i, j) \in \mathcal{M}_{h}}\left|f_{i+\alpha_{j}, j+\beta_{i}}\right|^{2}\right)^{1 / 2},
$$

where

$$
f_{i+\alpha_{j}, j+\beta_{i}}:=f\left(x_{i}+\alpha_{j} \Delta x, v_{j}+\beta_{i} \Delta v\right), \quad(i, j) \in\left[0, N_{x}\right] \times\left[0, N_{v}\right] .
$$

In the sequel, we fix an ending time $T$ and consider a uniform time discretization $\left\{t^{n}\right\}_{n \leq N_{T}}$ of the interval $[0, T]$ with a time step $\Delta t=t^{n+1}-t^{n}$. We shall find an approximation $f_{h}\left(t^{n}, x_{i}, v_{j}\right)$ for the exact distribution function $f\left(t^{n}, x_{i}, v_{j}\right)$ at each point $\left(x_{i}, v_{j}\right) \in \mathcal{M}_{h}$ and at time $t^{n}=n \Delta t$. The approximation function $f_{h}\left(t^{n}\right)$ is then evaluated at each point of $\mathbb{R}_{x} \times \mathbb{R}_{v}$ using the interpolation operator $\mathscr{R}_{h}$ defined on a uniform grid:

$$
\begin{aligned}
\mathscr{R}_{h}: L^{2}(\Omega) \cap \mathcal{P}(\Omega) & \longrightarrow L^{2}(\Omega) \cap \mathcal{P}(\Omega), \\
f & \longrightarrow \mathscr{R}_{h} f=\sum_{(i, j) \in \mathbb{Z} \times \mathbb{Z}} f_{i, j} \psi_{i, j},
\end{aligned}
$$

where $\left\{\psi_{i, j}\right\}_{(i, j) \in \mathbb{Z} \times \mathbb{Z}}$ are some basis functions. In order to deduce a convergent scheme, the interpolation operator $\mathscr{R}_{h}$ must satisfy some stability and high-order approximation properties which are detailed afterwards. We give some examples for $\mathscr{R}_{h}$ in section 5 .

3.2. The numerical scheme. Here we define the electric field operator for the real-valued function $g \in L^{1}([0, L] \times \mathbb{R})$, such that

$$
E(g, x)=\int_{0}^{L} K(x, y)\left(\int_{\mathbb{R}} g(y, v) d v-1\right),
$$

and the transport operators in the $x$-direction $\mathcal{T}_{1}$ and $\widetilde{\mathcal{T}}_{1}$ by

$$
\mathcal{T}_{1} f(t, x, v)=f(t, x-v \Delta t / 2, v), \quad f \in \mathscr{C}_{b}\left(0, T ; \mathscr{C}_{c, p e r_{x}}\left(\mathbb{R}_{x} \times \mathbb{R}_{v}\right)\right)
$$

and

$$
\widetilde{\mathcal{T}}_{1} f(t, x, v)=\mathscr{R}_{h} \circ \mathcal{T}_{1} f(t, x, v)=\mathscr{R}_{h} f(t, x-v \Delta t / 2, v) .
$$

Let $\widetilde{g}$ be a given function in $L^{1}([0, L] \times \mathbb{R})$, allowing us to define the transport operator in the $v$-direction $\mathcal{T}_{2}(\cdot, \cdot)$ that acts upon a function $g$ through

$$
\mathcal{T}_{2}(g, \widetilde{g})=g(x, v-E(\widetilde{g}, x) \Delta t) .
$$

For the convergence analysis we introduce three more operators $\mathcal{T}_{2}, \widetilde{\mathcal{T}}_{2}$, and $\widetilde{\mathcal{T}}_{2}^{\star}$ :

$$
\mathcal{T}_{2} g=\mathcal{T}_{2}(g, g), \quad \widetilde{\mathcal{T}}_{2} g=\mathscr{R}_{h} \circ \mathcal{T}_{2}\left(g, \mathcal{T}_{1} f\right), \quad \widetilde{\mathcal{T}}_{2}^{\star} g=\mathscr{R}_{h} \circ \mathcal{T}_{2}\left(g, \widetilde{\mathcal{T}}_{1} f_{h}\right) .
$$

The time discretization is based on a Strang splitting of the global transport operator into two transport operators in the configuration space and in the velocity space. These spaces have the associated ODEs which can be integrated analytically. Supposing that we know $f_{h}\left(t^{n}\right)$ at time $t^{n}$, the numerical scheme is decomposed into the following steps: 
S1: We compute a first half-backward advection in the $x$-direction of an increment $(v \Delta t / 2)$ : at each coordinate point $(x, v) \in \mathcal{M}_{h}, f_{h}\left(t^{n}, x-v \Delta t / 2, v\right)$ is evaluated. The new approximation after this first fractional time step is then given by

$$
f_{h}^{\dagger}\left(t^{n}\right)=\widetilde{\mathcal{T}}_{1} f_{h}\left(t^{n}\right)
$$

S2: The new approximation for the electric field from the previous solution at each coordinate point $(x, v) \in \mathcal{M}_{h}$ is

$$
E_{h}\left(t^{n+1 / 2}, x\right)=E\left(f_{h}^{\dagger}\left(t^{n}\right), x\right) .
$$

S3: We compute a backward advection in the $v$-direction of an increment $\left(\Delta t E\left(f_{h}^{\dagger}\left(t^{n}\right), x\right)\right.$ : at each point $(x, v) \in \mathcal{M}_{h} f_{h}^{\dagger}\left(t^{n}, x, v-E\left(f_{h}^{\dagger}\left(t^{n}\right), x\right) \Delta t\right)$ is evaluated. The new approximation after this second fractional step is then given by

$$
f_{h}^{\ddagger}\left(t^{n}\right)=\widetilde{\mathcal{T}}_{2}^{\star} f_{h}^{\dagger}\left(t^{n}\right) .
$$

S4: We repeat step (S1), and the new approximation at time $t^{n+1}$ is given by

$$
f_{h}\left(t^{n+1}\right)=\widetilde{\mathcal{T}}_{1} f_{h}^{\ddagger}\left(t^{n}\right) .
$$

The numerical schemes can be summarized as

$$
f_{h}\left(t^{n+1}, x, v\right)=\widetilde{\mathcal{T}}_{1} \circ \widetilde{\mathcal{T}}_{2}^{\star} \circ \widetilde{\mathcal{T}}_{1} f_{h}\left(t^{n}, x, v\right),
$$

with $f_{h}^{0}=\mathscr{R}_{h} f_{0}$ the discretization of the initial data $f_{0}$. The boundary conditions are $f_{h}^{n}(x+L, v)=f_{h}^{n}(x, v), \forall|v| \leq R, \forall x \in[0, L]$ in the $x$-direction and $f_{h}^{n}(x, v)=0$, $\forall|v|>R, \forall x \in[0, L]$ in the $v$-direction.

\section{Convergence analysis}

Here we state the main theorem which yields convergence and error estimates for our scheme.

Theorem 2. Assume that $f_{0} \in W_{c, p e r}^{m+1, \infty}\left(\mathbb{R}_{x} \times \mathbb{R}_{v}\right)$ is positive, periodic in $x$ with period L, and is compactly supported in velocity. In addition, we assume that the interpolation operator $\mathscr{R}_{h}$ satisfies the following properties:

i) Consistency and high-order accuracy: Let $m, p$, and $k$ be some integers such that $m \geq 0,1 \leq p \leq \infty$, and $0 \leq k \leq 1$. The following interpolation error estimate then holds:

$$
\| f-\left.\mathscr{R}_{h} f\right|_{W^{k, p}(\Omega)} \leq C h^{m+1-k}|f|_{W^{m+1, p}(\Omega)}, \quad \forall f \in W^{m+1, p}(\Omega) \cap \mathcal{P}(\Omega) .
$$

ii) Stability: Let $f$ belong to $\mathscr{C}(\Omega) \cap \mathcal{P}(\Omega)$. We then have

$$
\begin{aligned}
& c\|f\|_{L_{h}^{2}(\Omega)} \leq\left\|\mathscr{R}_{h} f\right\|_{L^{2}(\Omega)} \leq C\|f\|_{L_{h}^{2}(\Omega)}, \quad \text { where } c \text { and } C \text { are independent of } h, \\
& \text { and } \\
& \quad\left\|\mathscr{R}_{h} f\right\|_{L_{h}^{2}, \Delta_{h}^{\mathbf{0}, \boldsymbol{\beta}}} \leq\|f\|_{L_{h}^{2}(\Omega)}, \quad\left\|\mathscr{R}_{h} f\right\|_{L_{h}^{2}, \Delta_{h}^{\boldsymbol{\alpha}, \mathbf{o}}} \leq\|f\|_{L_{h}^{2}(\Omega)} .
\end{aligned}
$$

Therefore the numerical solution of the Vlasov-Poisson system $\left(f_{h}, E_{h}\right)$ computed by the numerical scheme presented in section 3.2 converges towards the solution 
$(f, E)$ of the periodic Vlasov-Poisson system, and there exists a constant $C=$ $C\left(\|f\|_{W^{2, \infty}\left(0, T ; W^{m+1, \infty}(\Omega)\right)}\right)$ independent of $\Delta t$ and $h$ such that

$$
\left\|f-f_{h}\right\|_{l^{\infty}\left(0, T ; L^{2}(\Omega)\right)}+\left\|E-E_{h}\right\|_{l^{\infty}\left(0, T ; L^{\infty}([0, L])\right)} \leq C\left(\Delta t^{2}+h^{m+1}+\frac{h^{m+1}}{\Delta t}\right) .
$$

Remark 1. As we have obtained error estimates in the $L^{2}$ norm, we would have preferred that the constant in the error estimates depend on the data, solely in the $H^{m}$ norm. Unfortunately, it seems that it is not possible to get rid of the $W^{m, \infty}$ norm because we have to estimate the product of the kind of electric field time distribution function when we estimate the coupling error (cf. Lemma 3). Nevertheless, in the adaptive case we expect that the dependence on the data is only in the $H^{m}$ norm because the threshold which determines the adaptive mesh could be estimated according to this latter norm. Therefore a weaker smoothness in $H^{m}$ could lead to a lower complexity in the case of an adaptive algorithm than in the uniform one (with $W^{m, \infty}$-norm data dependence).

4.1. Idea of the proof. In order to apply a discrete Grönwall inequality we express the global error at time $t^{n+1}$ by

$$
e^{n+1}=\left\|f\left(t^{n+1}, x, v\right)-f_{h}\left(t^{n+1}, x, v\right)\right\|_{L_{h}^{2}(\Omega)},
$$

according to $e^{n}$. We therefore decompose $f\left(t^{n+1}, x, v\right)-f_{h}\left(t^{n+1}, x, v\right)$ into

$$
\begin{aligned}
& f\left(t^{n+1}, x, v\right)-f_{h}\left(t^{n+1}, x, v\right) \\
& =\left(f\left(t^{n+1}, x, v\right)-\mathcal{T}_{1} \circ \mathcal{T}_{2} \circ \mathcal{T}_{1} f\left(t^{n}, x, v\right)\right) \\
& \quad+\left(\mathcal{T}_{1} \circ \mathcal{T}_{2} \circ \mathcal{T}_{1} f\left(t^{n}, x, v\right)-\widetilde{\mathcal{T}}_{1} \circ \widetilde{\mathcal{T}}_{2} \circ \widetilde{\mathcal{T}}_{1} f\left(t^{n}, x, v\right)\right) \\
& \quad+\left(\widetilde{\mathcal{T}}_{1} \circ \widetilde{\mathcal{T}}_{2} \circ \widetilde{\mathcal{T}}_{1}-\widetilde{\mathcal{T}}_{1} \circ \widetilde{\mathcal{T}}_{2}^{\star} \circ \widetilde{\mathcal{T}}_{1}\right) f\left(t^{n}, x, v\right) \\
& \quad+\left(\widetilde{\mathcal{T}}_{1} \circ \widetilde{\mathcal{T}}_{2}^{\star} \circ \widetilde{\mathcal{T}}_{1}\left(f\left(t^{n}, x, v\right)-f_{h}\left(t^{n}, x, v\right)\right)\right) .
\end{aligned}
$$

In order to evaluate $e^{n+1}$, we will first estimate the four terms on the right-hand side of this equation. These estimations are described in the following section.

4.2. A priori estimates. We begin with a lemma which states the error estimate for time discretization.

Lemma 1. Assume that $f \in W^{2, \infty}\left(0, T ; W_{c, p^{2} r_{x}}^{2, \infty}\left(\mathbb{R}_{x} \times \mathbb{R}_{v}\right)\right)$; then there exists $C$ such that

$$
\left\|f\left(t^{n+1}\right)-\mathcal{T}_{1} \circ \mathcal{T}_{2} \circ \mathcal{T}_{1} f\left(t^{n}\right)\right\|_{L_{h}^{2}(\Omega)} \leq C \Delta t^{3} .
$$

Proof. We have

$$
\begin{aligned}
& \left\|f\left(t^{n+1}\right)-\mathcal{T}_{1} \circ \mathcal{T}_{2} \circ \mathcal{T}_{1} f\left(t^{n}\right)\right\|_{L_{h}^{2}(\Omega)} \\
& \quad \leq C\left\|f\left(t^{n+1}\right)-\mathcal{T}_{1} \circ \mathcal{T}_{2} \circ \mathcal{T}_{1} f\left(t^{n}\right)\right\|_{L_{h}^{\infty}(\Omega)} \\
& \quad \leq C\left\|f\left(t^{n+1}\right)-\mathcal{T}_{1} \circ \mathcal{T}_{2} \circ \mathcal{T}_{1} f\left(t^{n}\right)\right\|_{L^{\infty}(\Omega)} \leq C \Delta t^{3} .
\end{aligned}
$$

This last inequality relies on the proof of the Lemma 5.3 in $[6$.

The next lemma gives estimates for the phase-space discretization error (15). 
Lemma 2. Assume that $f \in L^{\infty}\left(0, T ; W_{c, p e r}^{m+1, \infty}\left(\mathbb{R}_{x} \times \mathbb{R}_{v}\right)\right)$ and let $\mathscr{R}_{h}$ be an interpolation operator satisfying (12)-(14). Then there exists a constant $C$ such that

$$
\left\|\mathcal{T}_{1} \circ \mathcal{T}_{2} \circ \mathcal{T}_{1} f\left(t^{n}\right)-\widetilde{\mathcal{T}}_{1} \circ \widetilde{\mathcal{T}}_{2} \circ \widetilde{\mathcal{T}}_{1} f\left(t^{n}\right)\right\|_{L_{h}^{2}(\Omega)} \leq C h^{m+1} .
$$

Proof. First we note the following decomposition

(16) $\mathcal{T}_{1} \circ \mathcal{T}_{2} \circ \mathcal{T}_{1} f\left(t^{n}\right)-\widetilde{\mathcal{T}}_{1} \circ \widetilde{\mathcal{T}}_{2} \circ \widetilde{\mathcal{T}}_{1} f\left(t^{n}\right)$

$$
=\left(\mathcal{T}_{1}-\widetilde{\mathcal{T}}_{1}\right) \circ \mathcal{T}_{2} \circ \mathcal{T}_{1} f\left(t^{n}\right)+\widetilde{\mathcal{T}}_{1} \circ\left(\mathcal{T}_{2}-\widetilde{\mathcal{T}}_{2}\right) \circ \mathcal{T}_{1} f\left(t^{n}\right)+\widetilde{\mathcal{T}}_{1} \circ \widetilde{\mathcal{T}}_{2} \circ\left(\mathcal{T}_{1}-\widetilde{\mathcal{T}}_{1}\right) f\left(t^{n}\right) .
$$

Using the estimates (12)-(14), we obtain for the first term of the decomposition (16)

$$
\begin{aligned}
& \left\|\left(\mathcal{T}_{1}-\widetilde{\mathcal{T}}_{1}\right) \circ \mathcal{T}_{2} \circ \mathcal{T}_{1} f\left(t^{n}\right)\right\|_{L_{h}^{2}(\Omega)} \leq C\left\|\mathscr{R}_{h} \circ\left(\mathcal{T}_{1}-\widetilde{\mathcal{T}}_{1}\right) \circ \mathcal{T}_{2} \circ \mathcal{T}_{1} f\left(t^{n}\right)\right\|_{L^{2}(\Omega)} \\
& \leq C\left\|\left(I-\mathscr{R}_{h}\right) \circ \mathcal{T}_{1} \circ \mathcal{T}_{2} \circ \mathcal{T}_{1} f\left(t^{n}\right)\right\|_{L^{2}(\Omega)} \\
& \quad+C\left\|\left(\mathscr{R}_{h}-I\right) \circ \mathcal{T}_{1} \circ \mathcal{T}_{2} \circ \mathcal{T}_{1} f\left(t^{n}\right)\right\|_{L^{2}(\Omega)} \\
& \leq C\left(\|f\|_{L^{\infty}\left(0, T ; W^{m+1, \infty}(\Omega)\right)}\right) h^{m+1},
\end{aligned}
$$

and for the second term of (16)

$$
\begin{aligned}
& \left\|\widetilde{\mathcal{T}}_{1} \circ\left(\mathcal{T}_{2}-\widetilde{\mathcal{T}}_{2}\right) \circ \mathcal{T}_{1} f\left(t^{n}\right)\right\|_{L_{h}^{2}(\Omega)} \\
& \quad \leq\left\|\widetilde{\mathcal{T}}_{1} \circ \mathscr{R}_{h} \circ\left(\mathcal{T}_{2}-\widetilde{\mathcal{T}}_{2}\right) \circ \mathcal{T}_{1} f\left(t^{n}\right)\right\|_{L_{h}^{2}(\Omega)}+\left\|\widetilde{\mathcal{T}}_{1} \circ\left(I-\mathscr{R}_{h}\right) \circ \mathcal{T}_{2} \circ \mathcal{T}_{1} f\left(t^{n}\right)\right\|_{L_{h}^{2}(\Omega)} \\
& \quad \leq\left\|\left(\mathcal{T}_{2}-\widetilde{\mathcal{T}}_{2}\right) \circ \mathcal{T}_{1} f\left(t^{n}\right)\right\|_{L_{h}^{2}(\Omega)}+C\left\|\mathscr{R}_{h} \circ \mathcal{T}_{1} \circ\left(I-\mathscr{R}_{h}\right) \circ \mathcal{T}_{2} \circ \mathcal{T}_{1} f\left(t^{n}\right)\right\|_{L^{2}(\Omega)} .
\end{aligned}
$$

The first term on the right-hand side of this inequality can be treated as earlier. We therefore obtain

$$
\begin{aligned}
\| \widetilde{\mathcal{T}}_{1} \circ & \left(\mathcal{T}_{2}-\widetilde{\mathcal{T}}_{2}\right) \circ \mathcal{T}_{1} f\left(t^{n}\right) \|_{L_{h}^{2}(\Omega)} \\
\leq & C h^{m+1}+C\left\|\left(\mathscr{R}_{h}-I\right) \circ \mathcal{T}_{1} \circ\left(I-\mathscr{R}_{h}\right) \circ \mathcal{T}_{2} \circ \mathcal{T}_{1} f\left(t^{n}\right)\right\|_{L^{2}(\Omega)} \\
& +C\left\|\mathcal{T}_{1} \circ\left(I-\mathscr{R}_{h}\right) \circ \mathcal{T}_{2} \circ \mathcal{T}_{1} f\left(t^{n}\right)\right\|_{L^{2}(\Omega)} \\
\leq & C h^{m+1}+C h\left|\left(I-\mathscr{R}_{h}\right) \circ \mathcal{T}_{2} \circ \mathcal{T}_{1} f\left(t^{n}\right)\right|_{W^{1,2}(\Omega)} \\
& +C\left\|\left(I-\mathscr{R}_{h}\right) \mathcal{T}_{2} \circ \mathcal{T}_{1} f\left(t^{n}\right)\right\|_{L^{2}(\Omega)} \\
\leq & C\left(\|f\|_{L^{\infty}\left(0, T ; W^{m+1, \infty}(\Omega)\right)}\right) h^{m+1} .
\end{aligned}
$$

Similarly, we get for the third term of (16) the following estimate:

$$
\begin{aligned}
\left\|\widetilde{\mathcal{T}}_{1} \circ \widetilde{\mathcal{T}}_{2} \circ\left(\mathcal{T}_{1}-\widetilde{\mathcal{T}}_{1}\right) f\left(t^{n}\right)\right\|_{L_{h}^{2}(\Omega)} & \leq\left\|\widetilde{\mathcal{T}}_{2} \circ\left(\mathcal{T}_{1}-\widetilde{\mathcal{T}}_{1}\right) f\left(t^{n}\right)\right\|_{L_{h}^{2}(\Omega)} \\
& \leq C\left(\|f\|_{L^{\infty}\left(0, T ; W^{m+1, \infty}(\Omega)\right)}\right) h^{m+1},
\end{aligned}
$$

which completes the proof.

The next lemma gives estimates for the coupling error (17) between the Vlasov and Poisson equations.

Lemma 3. Assume that $f \in L^{\infty}\left(0, T ; W_{c, p e r}^{m+1, \infty}\left(\mathbb{R}_{x} \times \mathbb{R}_{v}\right)\right)$ and let $\mathscr{R}_{h}$ be an interpolation operator satisfying (12) -(14). Then there exists a constant $C$ such that

$$
\left\|\widetilde{\mathcal{T}}_{1} \circ \widetilde{\mathcal{T}}_{2} \circ \widetilde{\mathcal{T}}_{1} f\left(t^{n}\right)-\widetilde{\mathcal{T}}_{1} \circ \widetilde{\mathcal{T}}_{2}^{\star} \circ \widetilde{\mathcal{T}}_{1} f\left(t^{n}\right)\right\|_{L_{h}^{2}(\Omega)} \leq C \Delta t\left(e^{n}+h^{m+1}\right)+C h^{m+1}
$$


and

$$
\left\|E_{h}^{n+1 / 2}-\widetilde{E}^{n+1 / 2}\right\|_{L^{\infty}([0, L])} \leq C\left(e^{n}+h^{m+1}\right),
$$

where

$$
\widetilde{E}^{n+1 / 2}(x)=\int_{0}^{L} K(x, y)\left(\int_{\mathbb{R}} \mathcal{T}_{1} f(t, y, v) d v-1\right) d y
$$

and

$$
e^{n}=\left\|f\left(t^{n}\right)-f_{h}\left(t^{n}\right)\right\|_{L_{h}^{2}(\Omega)}
$$

Proof. We have

$$
\left(\widetilde{\mathcal{T}}_{2}-\widetilde{\mathcal{T}}_{2}^{\star}\right) g\left(t^{n}\right)=\mathscr{R}_{h}\left(g\left(t^{n}, x, v-\Delta t \widetilde{E}^{n+1 / 2}(x)\right)-g\left(t^{n}, x, v-\Delta t E_{h}^{n+1 / 2}(x)\right)\right)
$$

and

$$
\begin{gathered}
\left|g\left(t^{n}, x, v-\Delta t \widetilde{E}^{n+1 / 2}(x)\right)-g\left(t^{n}, x, v-\Delta t E_{h}^{n+1 / 2}(x)\right)\right| \\
\leq \Delta t\left|\widetilde{E}^{n+1 / 2}(x)-E_{h}^{n+1 / 2}(x)\right|\left\|\nabla g\left(t^{n}\right)\right\|_{L^{\infty}(Q) .}
\end{gathered}
$$

Using Cauchy-Schwarz inequality and the estimates (12)-(14), we get

$$
\begin{aligned}
&\left\|E_{h}^{n+1 / 2}-\widetilde{E}^{n+1 / 2}\right\|_{L^{2}([0, L])} \leq L \sqrt{Q(T)}\|K\|_{L^{\infty}}\left\|\widetilde{\mathcal{T}}_{1} f_{h}\left(t^{n}\right)-\mathcal{T}_{1} f\left(t^{n}\right)\right\|_{L^{2}(\Omega)} \\
& \leq L \sqrt{Q(T)}\|K\|_{L^{\infty}}\left\{\left\|\widetilde{\mathcal{T}}_{1}\left(f_{h}\left(t^{n}\right)-f\left(t^{n}\right)\right)\right\|_{L^{2}(\Omega)}+\left\|\widetilde{\mathcal{T}}_{1} f\left(t^{n}\right)-\mathcal{T}_{1} f\left(t^{n}\right)\right\|_{L^{2}(\Omega)}\right\} \\
& \leq C\left\|\mathscr{R}_{h} \circ \mathcal{T}_{1} \circ \mathscr{R}_{h}\left(f_{h}\left(t^{n}\right)-f\left(t^{n}\right)\right)\right\|_{L^{2}(\Omega)}+C\left\|\mathscr{R}_{h} \circ \mathcal{T}_{1} \circ\left(I-\mathscr{R}_{h}\right) f\left(t^{n}\right)\right\|_{L^{2}(\Omega)} \\
&+C h^{m+1}\left\|\mathcal{T}_{1} f\right\|_{L^{\infty}\left(0, T ; W^{m+1,2}(\Omega)\right)} \\
& \leq C e_{n}+C\left\|\mathcal{T}_{1} \circ\left(I-\mathscr{R}_{h}\right) f\left(t^{n}\right)\right\|_{L^{2}(\Omega)} \\
&+C\left\|\left(\mathscr{R}_{h}-I\right) \circ \mathcal{T}_{1} \circ\left(I-\mathscr{R}_{h}\right) f\left(t^{n}\right)\right\|_{L^{2}(\Omega)}+C h^{m+1} \\
& \leq C\left(e^{n}+h^{m+1}\right)+C h^{m+1}\left\|f\left(t^{n}\right)\right\|_{W^{m+1,2}(\Omega)}+C h\left|\left(I-\mathscr{R}_{h}\right) f\left(t^{n}\right)\right|_{W^{1,2}(\Omega)} \\
& \leq C\left(\|f\|_{L^{\infty}\left(0, T ; W^{m+1, \infty}(\Omega)\right)}\right)\left(e^{n}+h^{m+1}\right) .
\end{aligned}
$$

Using the Poisson equation, we also obtain

$$
\begin{aligned}
\left|E_{h}^{n+1 / 2}-\widetilde{E}^{n+1 / 2}\right|_{H^{1}([0, L])} & \leq \sqrt{Q(T)}\left\|\widetilde{\mathcal{T}}_{1} f_{h}\left(t^{n}\right)-\mathcal{T}_{1} f\left(t^{n}\right)\right\|_{L^{2}(\Omega)} \\
& \leq C\left(e^{n}+h^{m+1}\right) .
\end{aligned}
$$

As we have the continuous imbedding $H^{1} \hookrightarrow L^{\infty}$, the estimates (20) and (21) lead to (18). Hence, we have

$$
\begin{aligned}
\left\|\widetilde{\mathcal{T}}_{1} \circ\left(\widetilde{\mathcal{T}}_{2}-\widetilde{\mathcal{T}}_{2}^{\star}\right) \circ \widetilde{\mathcal{T}}_{1} f\left(t^{n}\right)\right\|_{L_{h}^{2}(\Omega)} & \leq\left\|\left(\mathcal{T}_{2}-\mathcal{T}_{2}^{\star}\right) \circ \widetilde{\mathcal{T}}_{1} f\left(t^{n}\right)\right\|_{L_{h}^{2}(\Omega)} \\
& \leq C\left\|\mathscr{R}_{h} \circ\left(\mathcal{T}_{2}-\mathcal{T}_{2}^{\star}\right) \circ \widetilde{\mathcal{T}}_{1} f\left(t^{n}\right)\right\|_{L^{2}(\Omega)}
\end{aligned}
$$


We note that we have the following decomposition:

$$
\begin{aligned}
\| \mathscr{R}_{h} \circ & \left(\mathcal{T}_{2}-\mathcal{T}_{2}^{\star}\right) \circ \widetilde{\mathcal{T}}_{1} f\left(t^{n}\right) \|_{L^{2}(\Omega)} \\
\leq & \left\|\left(\mathcal{T}_{2}-\mathcal{T}_{2}^{\star}\right) \circ \widetilde{\mathcal{T}}_{1} f\left(t^{n}\right)\right\|_{L^{2}(\Omega)} \\
& +\left\|\left(\mathscr{R}_{h}-I\right) \circ\left(\mathcal{T}_{2}-\mathcal{T}_{2}^{\star}\right) \circ\left(\mathscr{R}_{h}-I\right) \circ \mathcal{T}_{1} f\left(t^{n}\right)\right\|_{L^{2}(\Omega)} \\
& +\left\|\left(\mathscr{R}_{h}-I\right) \circ\left(\mathcal{T}_{2}-\mathcal{T}_{2}^{\star}\right) \circ \mathcal{T}_{1} f\left(t^{n}\right)\right\|_{L^{2}(\Omega)} .
\end{aligned}
$$

Using (19) we get for the first term in (22)

$$
\begin{aligned}
\left\|\left(\mathcal{T}_{2}-\mathcal{T}_{2}^{\star}\right) \circ \widetilde{\mathcal{T}}_{1} f\left(t^{n}\right)\right\|_{L^{2}(\Omega)} & \leq \Delta t\left\|E_{h}^{n+1 / 2}-\widetilde{E}^{n+1 / 2}\right\|_{L^{2}([0, L])}\left\|\nabla\left(\widetilde{\mathcal{T}}_{1} f\left(t^{n}\right)\right)\right\|_{L^{\infty}(\Omega)} \\
& \leq C \Delta t\left(e^{n}+h^{m+1}\right)\left\|\nabla\left(\widetilde{\mathcal{T}}_{1} f\left(t^{n}\right)\right)\right\|_{L^{\infty}(\Omega)} .
\end{aligned}
$$

Now we must show that the term $\left\|\nabla\left(\widetilde{\mathcal{T}}_{1} f\left(t^{n}\right)\right)\right\|_{L^{\infty}(\Omega)}$ is bounded.

$$
\begin{aligned}
\left\|\nabla\left(\widetilde{\mathcal{T}}_{1} f\left(t^{n}\right)\right)\right\|_{L^{\infty}(\Omega)} \leq & \left\|\nabla\left(\mathscr{R}_{h} f\left(t^{n}, x-v \Delta t / 2, v\right)\right)\right\|_{L^{\infty}(\Omega)} \\
\leq & \left\|\nabla\left[\left(\mathscr{R}_{h} f-f\right)\left(t^{n}, x-v \Delta t / 2, v\right)\right]\right\|_{L^{\infty}(\Omega)} \\
& +\left\|\nabla\left(f\left(t^{n}, x-v \Delta t / 2, v\right)\right)\right\|_{L^{\infty}(\Omega)} \\
\leq & h^{m}\|f\|_{L^{\infty}\left(0, T ; W^{m+1, \infty}(\Omega)\right)}+\|f\|_{L^{\infty}\left(0, T ; W^{m+1, \infty}(\Omega)\right)} \\
\leq & C\|f\|_{L^{\infty}\left(0, T ; W^{m+1, \infty}(\Omega)\right)} .
\end{aligned}
$$

For the second term of (22), we have

$$
\begin{aligned}
&\left\|\left(\mathscr{R}_{h}-I\right) \circ\left(\mathcal{T}_{2}-\mathcal{T}_{2}^{\star}\right) \circ\left(\mathscr{R}_{h}-I\right) \circ \mathcal{T}_{1} f\left(t^{n}\right)\right\|_{L^{2}(\Omega)} \\
& \leq C h\left|\left(\mathcal{T}_{2}-\mathcal{T}_{2}^{\star}\right) \circ\left(\mathscr{R}_{h}-I\right) \circ \mathcal{T}_{1} f\left(t^{n}\right)\right|_{W^{1,2}(\Omega)} \\
& \leq C h \Delta t\left(\left|\widetilde{E}^{n+1 / 2}\right|_{H^{1}}+\left|E_{h}^{n+1 / 2}\right|_{H^{1}}\right)\left|\left(\mathscr{R}_{h}-I\right) \circ \mathcal{T}_{1} f\left(t^{n}\right)\right|_{W^{1, \infty}(\Omega)} \\
& \quad+C h\left|\left(\mathscr{R}_{h}-I\right) \circ \mathcal{T}_{1} f\left(t^{n}\right)\right|_{W^{1,2}(\Omega)} \\
& \leq C\left(\|\left. f\right|_{L^{\infty}\left(0, T ; W^{m+1, \infty}(\Omega)\right)}\right)(1+\Delta t) h^{m+1} .
\end{aligned}
$$

In order to justify the last inequality we must show that $\left|\widetilde{E}^{n+1 / 2}\right|_{H^{1}}$ and $\left|E_{h}^{n+1 / 2}\right|_{H^{1}}$ are bounded. Indeed, the Poisson equation and (13)-(14) lead to

$$
\begin{aligned}
\left|\widetilde{E}^{n+1 / 2}\right|_{H^{1}} & \leq \sqrt{Q(T)}\left\|\mathcal{T}_{1} f\left(t^{n}\right)\right\|_{L^{2}(\Omega)} \\
& \leq \sqrt{Q(T)}\left\|f\left(t^{n}\right)\right\|_{L^{2}(\Omega)} \leq C\|f\|_{L^{\infty}\left(0, T ; L^{\infty}(\Omega)\right)}
\end{aligned}
$$

and

$$
\begin{aligned}
\left|E_{h}^{n+1 / 2}\right|_{H^{1}} & \leq \sqrt{Q(T)}\left\|\mathscr{R}_{h} \circ \mathcal{T}_{1} f_{h}\left(t^{n}\right)\right\|_{L^{2}(\Omega)} \\
& \leq C \sqrt{Q(T)}\left\|\mathcal{T}_{1} f_{h}\left(t^{n}\right)\right\|_{L_{h}^{2}(\Omega)} \leq C\left\|f_{h}\left(t^{n}\right)\right\|_{L_{h}^{2}(\Omega)} \leq C .
\end{aligned}
$$

This last estimate comes from the $L_{h}^{2}$-stability property of the scheme. Indeed, from (14) we have

$$
\begin{aligned}
\left\|f_{h}\left(t^{n+1}\right)\right\|_{L_{h}^{2}(\Omega)} & \leq\left\|\mathscr{R}_{h} \circ \mathcal{T}_{1} \circ \mathscr{R}_{h} \circ \mathcal{T}_{2}^{\star} \circ \mathscr{R}_{h} \circ \mathcal{T}_{1} f_{h}\left(t^{n}\right)\right\|_{L_{h}^{2}(\Omega)} \\
& \leq\left\|f_{h}\left(t^{n}\right)\right\|_{L_{h}^{2}(\Omega)} \leq\left\|f_{h}\left(t^{0}\right)\right\|_{L_{h}^{2}(\Omega)} \leq C .
\end{aligned}
$$


Now it remains to estimate the third term of (22). First, let us compute $\left|\left(\mathcal{T}_{2}-\mathcal{T}_{2}^{\star}\right) g\right|_{W^{1,2}(\Omega)}$. We have

$$
\partial_{x}\left[\left(\mathcal{T}_{2}-\mathcal{T}_{2}^{\star}\right) g(x, v)\right]=E_{1}-\Delta t\left(E_{2}+E_{3}\right)
$$

and

$$
\partial_{v}\left[\left(\mathcal{T}_{2}-\mathcal{T}_{2}^{\star}\right) g(x, v)\right]=E_{4}
$$

with

$$
\begin{aligned}
& E_{1}=\partial_{x} g\left(x, v-\Delta t \widetilde{E}^{n+1 / 2}(x)\right)-\partial_{x} g\left(x, v-\Delta t E_{h}^{n+1 / 2}(x)\right), \\
& E_{2}=\left(\partial_{x} \widetilde{E}^{n+1 / 2}(x)-\partial_{x} E_{h}^{n+1 / 2}(x)\right) \partial_{v} g\left(x, v-\Delta t E_{h}^{n+1 / 2}(x)\right), \\
& E_{3}=\partial_{x} \widetilde{E}^{n+1 / 2}(x)\left(\partial_{v} g\left(x, v-\Delta t \widetilde{E}^{n+1 / 2}(x)\right)-\partial_{v} g\left(x, v-\Delta t E_{h}^{n+1 / 2}(x)\right)\right), \\
& E_{4}=\partial_{v} g\left(x, v-\Delta t \widetilde{E}^{n+1 / 2}(x)\right)-\partial_{v} g\left(x, v-\Delta t E_{h}^{n+1 / 2}(x)\right) .
\end{aligned}
$$

Now, let $j \in\left\{\partial_{x} g, \partial_{v} g\right\}$. We then have

$$
\begin{aligned}
& \left(\int_{\Omega}\left|j\left(x, v-\Delta t \widetilde{E}^{n+1 / 2}(x)\right)-j\left(x, v-\Delta t E_{h}^{n+1 / 2}(x)\right)\right|^{2} d x d v\right)^{1 / 2} \\
& \quad=\Delta t\left(\int_{\Omega}\left|\int_{\widetilde{E}^{n+1 / 2}(x)}^{E_{h}^{n+1 / 2}(x)} \partial_{v} j(x, v-\Delta t s) d s\right|^{2} d x d v\right)^{1 / 2} \\
& \quad \leq C \sqrt{Q(T)} \Delta t\left|\partial_{v} j\right|_{L^{\infty}(\Omega)}\left(\int_{0}^{L}\left|\widetilde{E}^{n+1 / 2}(x)-E_{h}^{n+1 / 2}(x)\right|^{2} d x\right)^{1 / 2} \\
& \quad \leq C \Delta t\left(e^{n}+h^{m+1}\right)\left|\partial_{v} j\right|_{L^{\infty}(\Omega)} .
\end{aligned}
$$

Finally, using (21) and (23)-(26) we get

$$
\left|\left(\mathcal{T}_{2}-\mathcal{T}_{2}^{\star}\right) g\right|_{W^{1,2}(\Omega)} \leq C \Delta t\left(e^{n}+h^{m+1}\right)\left(|g|_{W^{1, \infty}(\Omega)}+|g|_{W^{2, \infty}(\Omega)}\right) .
$$

Consequently, from (27) we get the estimate for the third term of (22):

$$
\begin{aligned}
& \left\|\left(\mathscr{R}_{h}-I\right) \circ\left(\mathcal{T}_{2}-\mathcal{T}_{2}^{\star}\right) \circ \mathcal{T}_{1} f\left(t^{n}\right)\right\|_{L^{2}(\Omega)} \\
& \quad \leq C h\left|\left(\mathcal{T}_{2}-\mathcal{T}_{2}^{\star}\right) \circ \mathcal{T}_{1} f\left(t^{n}\right)\right|_{W^{1,2}(\Omega)} \\
& \quad \leq C h \Delta t\left(e^{n}+h^{m+1}\right)\left(\left|\mathcal{T}_{1} f\left(t^{n}\right)\right|_{W^{1, \infty}(\Omega)}+\left|\mathcal{T}_{1} f\left(t^{n}\right)\right|_{W^{2, \infty}(\Omega)}\right) \\
& \quad \leq C\left(\|f\|_{L^{\infty}\left(0, T ; W^{2, \infty}(\Omega)\right)}\right) h \Delta t\left(e^{n}+h^{m+1}\right),
\end{aligned}
$$

which completes the proof of the lemma.

Now, we state the lemma which gives the crucial estimate of the $L^{2}$-stability.

Lemma 4. Let $\mathscr{R}_{h}$ be an interpolation operator satisfying (12)-(14). We then get

$$
\left\|\widetilde{\mathcal{T}}_{1} \circ \widetilde{\mathcal{T}}_{2}^{\star} \circ \widetilde{\mathcal{T}}_{1}\left(f\left(t^{n}\right)-f_{h}\left(t^{n}\right)\right)\right\|_{L_{h}^{2}(\Omega)} \leq e_{n}+C h^{m+1} .
$$


Proof. The estimates (12)-(14) obviously leads to

$$
\begin{aligned}
\left\|\widetilde{\mathcal{T}}_{1} \circ \widetilde{\mathcal{T}}_{2}^{\star} \circ \widetilde{\mathcal{T}}_{1}\left(f\left(t^{n}\right)-f_{h}\left(t^{n}\right)\right)\right\|_{L_{h}^{2}(\Omega)} \\
\leq\left\|\mathscr{R}_{h} \circ \mathcal{T}_{1} \circ \mathscr{R}_{h} \circ \mathcal{T}_{2}^{\star} \circ \mathscr{R}_{h} \circ \mathcal{T}_{1}\left(f\left(t^{n}\right)-f_{h}\left(t^{n}\right)\right)\right\|_{L_{h}^{2}(\Omega)} \\
\leq\left\|\mathcal{T}_{1}\left(f\left(t^{n}\right)-f_{h}\left(t^{n}\right)\right)\right\|_{L_{h}^{2}(\Omega)} \\
\leq\left\|\mathcal{T}_{1} \circ \mathscr{R}_{h}\left(f\left(t^{n}\right)-f_{h}\left(t^{n}\right)\right)\right\|_{L_{h}^{2}(\Omega)}+\left\|\mathcal{T}_{1} \circ\left(I-\mathscr{R}_{h}\right) f\left(t^{n}\right)\right\|_{L_{h}^{2}(\Omega)} \\
\leq e_{n}+C\left\|\mathcal{T}_{1} \circ\left(I-\mathscr{R}_{h}\right) f\left(t^{n}\right)\right\|_{L^{2}(\Omega)} \\
\quad+C\left\|\left(\mathscr{R}_{h}-I\right) \circ \mathcal{T}_{1} \circ\left(I-\mathscr{R}_{h}\right) f\left(t^{n}\right)\right\|_{L^{2}(\Omega)} \\
\leq e_{n}+C h^{m+1}\left\|f\left(t^{n}\right)\right\|_{W^{m+1,2}(\Omega)}+C h\left|\left(I-\mathscr{R}_{h}\right) f\left(t^{n}\right)\right|_{W^{1,2}(\Omega)} \\
\leq e_{n}+C\left(\|f\|_{L^{\infty}\left(0, T ; W^{m+1, \infty}(\Omega)\right)}\right) h^{m+1},
\end{aligned}
$$

which ends the proof.

We can now come back to the proof of Theorem 2

Proof of Theorem 2. Using the estimates stated in Lemmata 1, 2, 3 and 4, we get

$$
e^{n+1} \leq(1+C \Delta t) e^{n}+C\left(\|f\|_{W^{2, \infty}\left(0, T ; W^{m+1, \infty}(\Omega)\right)}\right)\left(\Delta t^{3}+h^{m+1}+h^{m+1} \Delta t\right) .
$$

A discrete Grönwall inequality leads to

$$
e^{n+1} \leq \exp (C T) e^{0}+C\left(\|f\|_{W^{2, \infty}\left(0, T ; W^{m+1, \infty}(\Omega)\right)}\right)\left(\Delta t^{2}+\frac{h^{m+1}}{\Delta t}+h^{m+1}\right) .
$$

As $e^{0}$ is a fixed interpolation error, using (33) and (43) finally we get

$$
\begin{aligned}
\left\|f-f_{h}\right\|_{l_{\infty}\left(0, T ; L^{2}(\Omega)\right)} & \leq\left\|\mathscr{R}_{h}\left(f-f_{h}\right)\right\|_{l_{\infty}\left(0, T ; L^{2}(\Omega)\right)}+\left\|\mathscr{R}_{h} f-f\right\|_{l^{\infty}\left(0, T ; L^{2}(\Omega)\right)} \\
& \leq C \sup _{n}\left\{e^{n}\right\}+C h^{m+1}\|f\|_{L^{\infty}\left(0, T ; W^{m+1, \infty}(\Omega)\right)} \\
& \leq C\left(\|f\|_{W^{2, \infty}\left(0, T ; W^{m+1, \infty}(\Omega)\right)}\right)\left(\Delta t^{2}+\frac{h^{m+1}}{\Delta t}+h^{m+1}\right) .
\end{aligned}
$$

The electric field error estimate is obtained as shown in the end of the proof of the Main Theorem 5.1 in $[6]$.

Remark 2. We note that our convergence analysis can be extended to higher splitting formulae in order to reach the order $N \geq 3$ in time. To build up approximations of the order $N$ in time (cf. [16, 17, 27]), we consider splitting schemes of the form

$f(t+\Delta t)=\mathcal{T}_{x}^{\alpha: 1}(\Delta t) \circ \mathcal{T}_{v}^{\beta: 1}(\Delta t) \circ \cdots \circ \mathcal{T}_{x}^{\alpha_{i}}(\Delta t) \circ \mathcal{T}_{v}^{\beta_{i}}(\Delta t) \circ \cdots \circ \mathcal{T}_{x}^{\alpha_{k}}(\Delta t) \circ \mathcal{T}_{v}^{\beta_{k}}(\Delta t) f(t)$

where

$$
\mathcal{T}_{x}^{\alpha_{i}}(\Delta t)=\exp \left(\alpha_{i} \Delta t \mathcal{L}_{x}\right), \quad \mathcal{T}_{v}^{\beta_{i}}(\Delta t)=\exp \left(\beta_{i} \Delta t \mathcal{L}_{v}\right),
$$

with

$$
\mathcal{L}_{x}=-v \cdot \partial_{x}, \quad \mathcal{L}_{v}=-E \cdot \partial_{v} .
$$

The actions of $\mathcal{T}_{x}^{\alpha_{i}}(\Delta t)$ and $\mathcal{T}_{v}^{\beta_{i}}(\Delta t)$ on the function $f(x, v)$ are, respectively,

$$
\mathcal{T}_{x}^{\alpha_{i}}(\Delta t) f(x, v)=f\left(x-\alpha_{i} v \Delta t, v\right), \quad \mathcal{T}_{v}^{\alpha_{i}}(\Delta t) f(x, v)=f\left(x, v-\beta_{i} E(t, x) \Delta t\right) .
$$


For example, if $\mathcal{T}_{2 n}(\Delta t)$, a $2 n$ th-order approximation of the global transport operator, is known, then a $(2 n+2)$ th-order approximation is obtained as follows:

$$
\begin{aligned}
& \mathcal{T}_{2 n+2}(\Delta t)=\mathcal{T}_{2 n}\left(\alpha_{n} \Delta t\right) \circ \mathcal{T}_{2 n}\left(\beta_{n} \Delta t\right) \circ \mathcal{T}_{2 n}\left(\alpha_{n} \Delta t\right) \\
& \text { with } \alpha_{n}=\frac{1}{2-2^{1 /(2 n+1)}} \text { and } \beta_{n}=\frac{1}{1-2^{2 n /(2 n+1)}} .
\end{aligned}
$$

\section{Some EXAmples FOR $\mathscr{R}_{h}$}

In this section we give two examples illustrating the interpolation operator $\mathscr{R}_{h}$. The first one is based on the symmetric Lagrange interpolation. The other one is built on B-splines. The Lagrange interpolation has the advantage of being local, so that it is well-suited for parallel computing. Nevertheless, it is more diffusive than B-splines. For example, in order to get the same rate of diffusion of the cubic B-splines a 9th order Lagrange interpolation must be used. This has been illustrated numerically in [15]. On the other hand, the B-splines interpolation is global, as a linear system involving all the points of computation domain should be solved. The B-splines interpolation has been chosen in many numerical applications 2, 13, 24. Hereafter, for each interpolation operator we explain its construction, recalling its useful properties before proving the crucial estimates (12)-(14). Note that in Theorem 2 we have given a general criteria on $\mathscr{R}_{h}$ which can be used for any reconstruction.

5.1. Symmetric Lagrange interpolation. Letting $x_{i+1 / 2}=\left(x_{i}+x_{i+1}\right) / 2$, we define the cells $C_{i+1 / 2, j+1 / 2}$ and $C_{i, j}$ by

$$
C_{i+1 / 2, j+1 / 2}=\left(x_{i}, x_{i+1}\right) \times\left(v_{j}, v_{j+1}\right), \quad C_{i, j}=\left(x_{i-1 / 2}, x_{i+1 / 2}\right) \times\left(v_{j-1 / 2}, v_{j+1 / 2}\right) .
$$

We then introduce the characteristic functions $\chi_{i+1 / 2, j+1 / 2}$ and $\chi_{i, j}$ defined by

$$
\chi_{i+1 / 2, j+1 / 2}(x, v)=\left\{\begin{array}{l}
1 \text { if }(x, v) \in C_{i+1 / 2, j+1 / 2}, \\
0 \text { otherwise }
\end{array}\right.
$$

and

$$
\chi_{i, j}(x, v)=\left\{\begin{array}{l}
1 \text { if }(x, v) \in C_{i, j} \\
0 \text { otherwise }
\end{array}\right.
$$

In one dimension, if $m$ is odd, we can then define the symmetric $m+1$ interpolation points on $\left(x_{i}, x_{i+1}\right)$ by

$$
x_{i-(m-1) / 2}, \ldots, x_{i+(m+1) / 2},
$$

and if $m$ is even, the $m+1$ interpolation points on $\left(x_{i-1 / 2}, x_{i+1 / 2}\right)$ are then

$$
x_{i-m / 2}, \ldots, x_{i+m / 2},
$$

which are also symmetric with respect to the interval $\left(x_{i-1 / 2}, x_{i+1 / 2}\right)$.

We denote by $\left\{\ell_{k, \Delta x}^{i}\right\}_{k \in\{0, \ldots, m\}}$ the Lagrange basis associated to the interval $\left(x_{i}, x_{i+1}\right)\left(\operatorname{resp} .\left(x_{i-1 / 2}, x_{i+1 / 2}\right)\right)$ :

$$
\ell_{k, \Delta_{x}}^{i}(z)=\prod_{\substack{s=0 \\ s \neq k}}^{s=m} \frac{\left(z-z_{s}\right)}{\left(z_{k}-z_{s}\right)},
$$

with the interpolation points $z_{s}=x_{i-(m-1) / 2}+s \Delta x$ (respectively $z_{s}=x_{i-m / 2}+$ $s \Delta x)$. Similarly we define $\ell_{k, \Delta v}^{j}$. Now, we define the interpolation operator $\mathscr{R}_{h}$ as 
follows, and consider a function $g$ defined on the grid $\mathcal{M}_{h}$. If $m$ is odd, we then define $\mathscr{R}_{h}$ as

$$
\mathscr{R}_{h} g(x, v)=\sum_{i \in \mathbb{Z}} \sum_{j \in \mathbb{Z}} \mathscr{R}_{h}^{i+1 / 2, j+1 / 2} g(x, v) \chi_{i+1 / 2, j+1 / 2}(x, v),
$$

where

$$
\begin{aligned}
\mathscr{R}_{h}^{i+1 / 2, j+1 / 2} g(x, v) & =\mathscr{R}_{h} g(x, v)_{\left.\right|_{C_{i+1 / 2, j+1 / 2}}} \\
& =\sum_{k=i-(m-1) / 2}^{i+(m+1) / 2} \sum_{l=j-(m-1) / 2}^{j+(m+1) / 2} g_{k, l} \ell_{k, \Delta x}^{i}(x) \ell_{l, \Delta v}^{j}(v) .
\end{aligned}
$$

If $m$ is even, we then define $\mathscr{R}_{h}$ as

$$
\mathscr{R}_{h} g(x, v)=\sum_{i \in \mathbb{Z}} \sum_{j \in \mathbb{Z}} \mathscr{R}_{h}^{i, j} g(x, v) \chi_{i, j}(x, v),
$$

where

$$
\mathscr{R}_{h}^{i, j} g(x, v)=\mathscr{R}_{h} g(x, v)_{\left.\right|_{C_{i, j}}}=\sum_{k=i-m / 2}^{i+m / 2} \sum_{l=j-m / 2}^{j+m / 2} g_{k, l} \ell_{k, \Delta x}^{i}(x) \ell_{l, \Delta v}^{j}(v)
$$

with $g_{k, l}=g\left(x_{k \bmod N_{x}+1}, v_{l \bmod N_{v}+1}\right)$.

The interpolation operator $\mathscr{R}_{h}$ satisfies the following interpolation error estimate (see [20]):

$$
\begin{aligned}
\left\|f-\mathscr{R}_{h} f\right\|_{W^{k, p}(\Omega)} \leq C h^{m+1-k}|f|_{W^{m+1, p}(\Omega)}, \quad f \in W^{m+1, p}(\Omega) \cap \mathcal{P}(\Omega), \\
k=0,1, \quad m \geq 0, \quad 1 \leq p \leq \infty
\end{aligned}
$$

5.1.1. Proof of the estimate (14). The first step of this proof consists in computing $\left\|\mathscr{R}_{h} f\right\|_{L_{h}^{2}, \Delta_{h}^{\boldsymbol{\alpha}, \mathbf{o}}}$ and $\left\|\mathscr{R}_{h} f\right\|_{L_{h}^{2}, \Delta_{h}^{\mathbf{0}, \boldsymbol{\beta}}}$. We recall that $\mathbf{N}=\left(N_{x}, N_{v}\right)$ and $\mathbf{z}_{k, l}=\left(x_{k}, v_{l}\right)$. The Fourier series decomposition of $f_{k, l}$ is

$$
f_{k, l}=\frac{1}{|\Omega|^{1 / 2}} \sum_{|\boldsymbol{\omega}| \leq \mathbf{N} / 2} \widehat{f}(\boldsymbol{\omega}) e^{i\left\langle\mathbf{k}(\boldsymbol{\omega}), \mathbf{z}_{k, l}\right\rangle}
$$

Introducing this formula in the definition of the interpolation operator $\mathscr{R}_{h}$ represented by the equations (29) -(32) and using the notations

$$
\begin{gathered}
p(i)=\left\{\begin{array}{rl}
i & \text { if } m \text { is even, } \\
i+1 / 2 & \text { if } m \text { is odd, } \\
m / 2 & \text { if } m \text { is even, }
\end{array} \quad q(i)=\left\{\begin{aligned}
i & \text { if } m \text { is even, } \\
i+1 / 2 & \text { if } m \text { is odd }
\end{aligned}\right.\right. \\
d_{b}(m)=\left\{\begin{aligned}
m / 2 & \text { if } m \text { is even, } \\
(m-1) / 2 & \text { if } m \text { is odd, }
\end{aligned}\right.
\end{gathered}
$$


we obtain

$$
\begin{aligned}
\left(\mathscr{R}_{h} f\right)_{i+\alpha_{j}, j}= & \frac{1}{|\Omega|^{1 / 2}} \sum_{|\boldsymbol{\omega}| \leq \mathbf{N} / 2} \sum_{k=i-d_{b}(m)}^{i+d_{e}(m)} \sum_{l=j-d_{b}(m)}^{j+d_{e}(m)} \\
& \times \widehat{f}(\boldsymbol{\omega}) \ell_{k}\left(x_{i}+\alpha_{j} \Delta x\right) \ell_{l}\left(v_{j}\right) e^{i\left\langle\mathbf{k}(\boldsymbol{\omega}), \mathbf{z}_{k, l}\right\rangle} \\
= & \frac{1}{|\Omega|^{1 / 2}} \sum_{|\boldsymbol{\omega}| \leq \mathbf{N} / 2} \sum_{k=i-d_{b}(m)}^{i+d_{e}(m)} \widehat{f}(\boldsymbol{\omega}) \ell_{k}\left(x_{i}+\alpha_{j} \Delta x\right) e^{i\left\langle\mathbf{k}(\boldsymbol{\omega}), \mathbf{z}_{k, j}\right\rangle} \\
= & \frac{1}{|\Omega|^{1 / 2}} \sum_{|\boldsymbol{\omega}| \leq \mathbf{N} / 2} \widehat{f}(\boldsymbol{\omega}) \varrho\left(\alpha_{j}, \omega_{x}\right) e^{-i\left\langle\mathbf{k}(\boldsymbol{\omega}),\left(d_{b}, 0\right)\right\rangle} e^{i\left\langle\mathbf{k}(\boldsymbol{\omega}), \mathbf{z}_{i, j}\right\rangle}
\end{aligned}
$$

where

$$
\varrho\left(\alpha_{j}, \omega_{x}\right)=\sum_{k=0}^{m} \ell_{k}\left(\left(d_{b}(m)+\alpha_{j}\right) \Delta x\right) e^{i k_{x}\left(\omega_{x}\right) x_{k}} .
$$

Hence,

$$
\begin{aligned}
&\left\|\mathscr{R}_{h} f\right\|_{L_{h}^{2}, \Delta_{h}^{\alpha, 0}}^{\alpha}= \Delta x \Delta v \sum_{i=0}^{N_{x}} \sum_{j=0}^{N_{v}}\left(\mathscr{R}_{h} f\right)_{i+\alpha_{j}, j}{\overline{\left(\mathscr{R}_{h} f\right)_{i+\alpha_{j}, j}}}_{=} \frac{\Delta x \Delta v}{|\Omega|} \sum_{i=0}^{N_{x}} \sum_{j=0}^{N_{v}} \sum_{|\boldsymbol{\omega}| \leq \mathbf{N} / 2} \sum_{\left|\boldsymbol{\omega}^{\prime}\right| \leq \mathbf{N} / 2} \widehat{f}(\boldsymbol{\omega}) \overline{\widehat{f}\left(\boldsymbol{\omega}^{\prime}\right)} \varrho\left(\alpha_{j}, \omega_{x}\right) \overline{\varrho\left(\alpha_{j}, \omega_{x}^{\prime}\right)} \\
& \times e^{-i\left\langle\mathbf{k}(\boldsymbol{\omega})-\mathbf{k}\left(\boldsymbol{\omega}^{\prime}\right),\left(d_{b}, 0\right)\right\rangle} e^{i\left\langle\mathbf{k}(\boldsymbol{\omega})-\mathbf{k}\left(\boldsymbol{\omega}^{\prime}\right), \mathbf{z}_{i, j}\right\rangle} .
\end{aligned}
$$

Since

$$
\frac{\Delta x}{|L|} \sum_{i=0}^{N_{x}} e^{i\left(k_{x}\left(\omega_{x}\right)-k_{x}\left(\omega_{x}^{\prime}\right)\right) x_{i}}=\delta_{\omega_{x}, \omega_{x}^{\prime}},
$$

it follows that

$$
\begin{aligned}
& \left\|\mathscr{R}_{h} f\right\|_{L_{h}^{2}, \Delta_{h}^{\alpha, \mathbf{0}}}^{2} \\
& \quad=\frac{\Delta v}{|2 R|} \sum_{j=0}^{N_{v}} \sum_{|\boldsymbol{\omega}| \leq \mathbf{N} / 2} \sum_{\left|\omega_{v}^{\prime}\right| \leq N_{v} / 2} \widehat{f}(\boldsymbol{\omega}) \overline{\hat{f}\left(\omega_{x}, \omega_{v}^{\prime}\right)}\left|\varrho\left(\alpha_{j}, \omega_{x}\right)\right|^{2} e^{i\left(k_{v}\left(\omega_{v}\right)-k_{v}\left(\omega_{v}^{\prime}\right)\right) v_{j}} .
\end{aligned}
$$

Since

$$
\frac{\Delta v}{|2 R|} \sum_{j=0}^{N_{v}} e^{i\left(k_{v}\left(\omega_{v}\right)-k_{v}\left(\omega_{v}^{\prime}\right)\right) v_{j}}=\delta_{\omega_{v}, \omega_{v}^{\prime}}
$$

we get

$$
\begin{aligned}
\left\|\mathscr{R}_{h} f\right\|_{L_{h}^{2}, \Delta_{h}^{\alpha, 0}}^{2} & \leq \sup \left\{\left|\varrho\left(\alpha, \omega_{x}\right)\right|^{2},\left|\omega_{x}\right| \leq N_{x} / 2,0 \leq \alpha \leq 1\right\} \\
& \times \frac{\Delta v}{|2 R|} \sum_{j=0}^{N_{v}} \sum_{|\boldsymbol{\omega}| \leq \mathbf{N} / 2} \sum_{\left|\omega_{v}^{\prime}\right| \leq N_{v} / 2} \widehat{f}(\boldsymbol{\omega}) \overline{\widehat{f}\left(\omega_{x}, \omega_{v}^{\prime}\right)} e^{i\left(k_{v}\left(\omega_{v}\right)-k_{v}\left(\omega_{v}^{\prime}\right)\right) v_{j}} \\
& \leq \sup \left\{\left|\varrho\left(\alpha, \omega_{x}\right)\right|^{2},\left|\omega_{x}\right| \leq N_{x} / 2,0 \leq \alpha \leq 1\right\} \sum_{|\boldsymbol{\omega}| \leq \mathbf{N} / 2}|\widehat{f}(\boldsymbol{\omega})|^{2} \\
& \leq \sup \left\{\left|\varrho\left(\alpha, \omega_{x}\right)\right|^{2},\left|\omega_{x}\right| \leq N_{x} / 2,0 \leq \alpha \leq 1\right\}\|f\|_{L_{h}^{2}(\Omega)}^{2} .
\end{aligned}
$$


Similarly, we obtain

$$
\left\|\mathscr{R}_{h} f\right\|_{L_{h}^{2}, \Delta_{h}^{\mathbf{0}, \boldsymbol{\beta}}}^{2} \leq \sup \left\{\left|\varrho\left(\beta, \omega_{v}\right)\right|^{2},\left|\omega_{v}\right| \leq N_{v} / 2,0 \leq \beta \leq 1\right\}\|f\|_{L_{h}^{2}(\Omega)}^{2} .
$$

The second step is to show that

$$
\sup \left\{\left|\varrho\left(\alpha, \omega_{x}\right)\right|^{2}, 0 \leq \alpha \leq 1,\left|\omega_{x}\right| \leq N_{x} / 2\right\} \leq 1
$$

and

$$
\sup \left\{\left|\varrho\left(\beta, \omega_{v}\right)\right|^{2}, \quad 0 \leq \beta \leq 1,\left|\omega_{v}\right| \leq N_{v} / 2\right\} \leq 1,
$$

where

$$
\varrho\left(\alpha, \omega_{x}\right)=\sum_{k=0}^{m} \ell_{k}\left(\left(d_{b}+\alpha\right) \Delta x\right) e^{i k_{x} x_{k}} \quad \text { and } \quad \varrho\left(\beta, \omega_{v}\right)=\sum_{l=0}^{m} \ell_{l}\left(\left(d_{b}+\beta\right) \Delta v\right) e^{i k_{v} v_{l}} .
$$

Without loss of generality, we can suppose that $L=2 R=2 \pi$ and $\Delta x=\Delta v=\Delta y$, so as to prove that $\sup \{|\varrho(y, \omega)|, y \in[-\Delta y(m-1) / 2, \Delta y(m+1) / 2], \omega \in \mathbb{Z}\} \leq 1$, where $\varrho(y, \omega)=\sum_{k=0}^{m} \ell_{k}(y) e^{i \omega y_{k}}$. We set $\theta=\omega \Delta y$ and $y=\eta \Delta y$, where $0 \leq \eta \leq m$. Since $\varrho(\eta, \theta)$ is periodic in $\theta$, we must now show that

$$
\sup \{|\varrho(\eta, \theta)|, \eta \in[(m-1) / 2,(m+1) / 2], \theta \in[0,2 \pi]\} \leq 1 .
$$

Finally, with the change of variables $\xi=1-\cos \theta$ and $\zeta=\eta-m / 2$, we obtain the following lemma which implies (37).

\section{Lemma 5.}

$$
\begin{aligned}
\left|\varrho_{2 n}^{\theta}(\zeta)\right|^{2}= & \left|\varrho_{2 n}(\zeta, \xi)\right|^{2} \\
= & 1-\zeta^{2}\left(1-\zeta^{2}\right) \cdots\left(n^{2}-\zeta^{2}\right) \xi^{n+1} \\
& {\left[c_{0}+c_{1} \xi\left(1-\zeta^{2}\right)+\cdots+c_{n-1} \xi^{n-1}\left(1-\zeta^{2}\right) \cdots\left((n-1)^{2}-\zeta^{2}\right)\right], } \\
& m=2 n, \\
\left|\varrho_{2 n-1}^{\theta}(\zeta)\right|^{2}= & \left|\varrho_{2 n-1}(\zeta, \xi)\right|^{2} \\
= & 1-\left(\left(\frac{1}{2}\right)^{2}-\zeta^{2}\right) \cdots\left(\left(\frac{2 n-1}{2}\right)^{2}-\zeta^{2}\right) \xi^{n} \\
& {\left[d_{0}+d_{1} \xi\left(\left(\frac{1}{2}\right)^{2}-\zeta^{2}\right)+\cdots+d_{n-1} \xi^{n-1}\left(\left(\frac{1}{2}\right)^{2}-\zeta^{2}\right) \cdots\left(\left(\frac{2 n-3}{2}\right)^{2}-\zeta^{2}\right)\right], } \\
& m=2 n+1,
\end{aligned}
$$

with

$$
c_{i}=\frac{2^{n+i+1}}{(2 n) !(2 i+1) !(n+1+i)}>0 \quad \text { and } \quad d_{i}=\frac{2^{n+i}}{(2 n-1) !(2 i) !(n+i)}>0 .
$$

Proof. The proofs of Theorems 3 and 4 are given in Appendix $\mathrm{A}$ 
5.1.2. Proof of the estimate (13). We suppose that $m$ is even (the proof is similar when $m$ is odd). We use a change of variable in the following computation:

$$
\begin{aligned}
\int_{C_{i, j}}\left|\mathscr{R}_{h} f\right|^{2} d x d v & =\int_{C_{i, j}}\left|\mathscr{R}_{h}^{i, j} f(x, v)\right|^{2} d x d v \\
& =\int_{C_{i, j}}\left|\sum_{|(k, l)| \leq \mathbf{m} / \mathbf{2}} f_{i+k, j+l} \ell_{i+k, \Delta x}^{i}(x) \ell_{j+l, \Delta v}^{j}(v)\right|^{2} d x d v \\
& =\Delta x \Delta v \int_{[-1 / 2,1 / 2]^{2}}\left|\sum_{|(k, l)| \leq \mathbf{m} / \mathbf{2}} f_{i+k, j+l} \ell_{k, 1}^{0}(x) \ell_{l, 1}^{0}(v)\right|^{2} d x d v \\
& =: \Delta x \Delta v G\left(f_{i-m / 2, j-m / 2}, \ldots, f_{i+m / 2, j+m / 2}\right) .
\end{aligned}
$$

The quantity $G$ is the square of a norm over $\mathbb{R}^{m+1}$, which is thus equivalent to the euclidian one, with the constants $c$ and $C$ depending on $m$, but not on $h$. We thus obtain

$$
\begin{aligned}
\int_{\Omega}\left|\mathscr{R}_{h} f\right|^{2} & \leq \sum_{(i, j)=\mathbf{0}}^{\mathbf{N}} \int_{C_{i, j}}\left|\mathscr{R}_{h} f\right|^{2} d x d v \leq C^{2} \Delta x \Delta v \sum_{(i, j)=\mathbf{0}}^{\mathbf{N}} \sum_{|(k, l)| \leq \mathbf{m} / \mathbf{2}}\left|f_{i+k, j+l}\right|^{2} \\
& \leq C^{2} \Delta x \Delta v(2 m+1)^{2} \sum_{(i, j)=\mathbf{0}}^{\mathbf{N}}\left|f_{i, j}\right|^{2}
\end{aligned}
$$

and the converse is also true, since we have

$$
\left|f_{i, j}\right|^{2} \leq \sum_{|(k, l)| \leq \mathbf{m} / \mathbf{2}}\left|f_{i+k, j+l}\right|^{2} \leq \frac{1}{c^{2}} \int_{C_{i, j}}\left|\mathscr{R}_{h} f\right|^{2} d x d v .
$$

5.2. B-splines interpolation. In this section we define the space of B-splines of order $m+1$ and the approximation space $Y_{h}$. Letting $m$ and $r$ be two positive integers, we define $\mathcal{B}_{m+1, \Delta_{x}}$, the linear space of the B-spline functions of order $m+1$ on $\mathbb{R}_{x}$, as

$$
\mathcal{B}_{m+1, \Delta_{x}}=\left\{s(x) \in \mathscr{C}^{m-1}(\mathbb{R}), \quad D^{m+1} s(x)=0, \forall x \in\left(x_{i}, x_{i+1}\right), \forall i \in \mathbb{Z}\right\},
$$

when $m+1$ is even, and as

$$
\mathcal{B}_{m+1, \Delta_{x}}=\left\{s(x) \in \mathscr{C}^{m-1}(\mathbb{R}), \quad D^{m+1} s(x)=0, \forall x \in\left(x_{i-1 / 2}, x_{i+1 / 2}\right), \forall i \in \mathbb{Z}\right\},
$$

when $m+1$ is odd.

Similarly we define $\mathcal{B}_{r+1, \Delta_{v}}$, the space of B-spline functions of order $r+1$ on $\mathbb{R}_{v}$. The space of B-spline functions in two dimensions is therefore defined as the tensor product of the spaces $\mathcal{B}_{m+1, \Delta_{x}}$ and $\mathcal{B}_{r+1, \Delta_{v}}$ :

$$
\begin{aligned}
\mathcal{B}_{m+1, r+1, \Delta_{x}, \Delta_{v}} & =\mathcal{B}_{m+1, \Delta_{x}} \otimes \mathcal{B}_{r+1, \Delta_{x}} \\
& =\left\{s(x, v)=s_{1}(x) s_{2}(v): s_{1} \in \mathcal{B}_{m+1, \Delta_{x}}, s_{2} \in \mathcal{B}_{r+1, \Delta_{v}}\right\} .
\end{aligned}
$$

Let us note that $\mathcal{S}_{m+1, r+1, \Delta_{x}, \Delta_{v}} \subset W^{k, p}\left(\mathbb{R}^{2}\right)$ with $k=\min (r, m)$ and $1 \leq p \leq \infty$. If we suppose that $r=m$, then $\mathcal{B}_{m+1, h}$ denotes the two-dimensional B-spline functions space of order $m+1$. Hence, the interpolation operator $\mathscr{R}_{h}$ is defined by

$$
\mathscr{R}_{h} f=\sum_{i \in \mathbb{Z}} \sum_{j \in \mathbb{Z}} \gamma_{i, j}(f) B_{m+1}(x / \Delta x-i) B_{m+1}(v / \Delta v-j)
$$


where the unidimensional B-spline $B_{m}$ of order $m$ is recursively defined by

$$
B_{m}(\cdot)=(\underbrace{B * \cdots * B}_{m \text { times }})(\cdot)=\int B_{m-1}(\cdot-u) B(u) d u
$$

with

$$
B_{1}(u)=B(u)= \begin{cases}1 & -1 / 2 \leq u \leq 1 / 2 \\ 0 & \text { elsewhere }\end{cases}
$$

The coefficients $\gamma_{i, j}(f)$ are solutions of the linear system

$$
f_{i, j}=\sum_{k, l} \gamma_{k, l}(f) B_{m+1}(i-k) B_{m+1}(j-l),
$$

$(i, j) \in\left[0, N_{x}\right] \times\left[0, N_{v}\right]$, and $\gamma_{i, j}(f):=\gamma_{i \bmod N_{x}+1, j \bmod N_{v}+1}(f)$. Now, we recall some useful properties on B-splines interpolation.

i)

$$
\mathcal{B}_{m+1, h}=\operatorname{Span}\left\{B_{m+1}(\cdot / \Delta x-i) B_{m+1}(\cdot / \Delta v-j) ; i \in \mathbb{Z}, j \in \mathbb{Z}\right\} .
$$

ii)

$$
\mathcal{B}_{m+1, h} \subset W^{m, p}, \quad 1 \leq p \leq \infty, \quad 0 \leq k \leq m .
$$

iii) Stability:

$$
\left\|\mathscr{R}_{h} f\right\|_{L^{p}(\Omega)} \leq C\|f\|_{L^{p}(\Omega)}, \quad \forall f \in L^{p}(\Omega) \cap \mathcal{P}(\Omega), \quad 1 \leq p \leq \infty .
$$

iv) Consistency and optimal accuracy: for $1 \leq p \leq \infty$ and $0 \leq k \leq m$,

$$
\left\|\mathscr{R}_{h} f-f\right\|_{W^{k, p}(\Omega)} \leq C h^{m+1-k}|f|_{W^{m+1, p}(\Omega)}, \quad \forall f \in W^{m+1, p}(\Omega) \cap \mathcal{P}(\Omega) .
$$

v) As the matrix $\left[\sum_{k, l} B_{m+1}(i-k) B_{m+1}(j-l)\right]_{i, j=0}^{N_{x}, N_{v}}$ is positive and definite, a unique solution of the linear system (39) exists.

vi)

$$
\sum_{i} B_{m}(\cdot / h-i)=1, \quad \int B_{m}(u) d u=1
$$

vii) The B-spline $B_{m, i}(\cdot)=B_{m}(\cdot / h-i)$ is constructed on the points

$$
\left\{x_{i-m / 2}, \ldots, x_{i+m / 2}\right\} .
$$

5.2.1. Proof of the estimate (14). By substituting

$$
f_{i, j}=\frac{1}{|\Omega|^{1 / 2}} \sum_{|\boldsymbol{\omega}| \leq \mathbf{N} / 2} \widehat{f}(\boldsymbol{\omega}) e^{i\left\langle\mathbf{k}(\boldsymbol{\omega}), \mathbf{z}_{i, j}\right\rangle} \quad \text { and } \quad \gamma_{k, l}=\frac{1}{|\Omega|^{1 / 2}} \sum_{|\boldsymbol{\omega}| \leq \mathbf{N} / 2} \widehat{\gamma}(\boldsymbol{\omega}) e^{i\left\langle\mathbf{k}(\boldsymbol{\omega}), \mathbf{z}_{k, l}\right\rangle}
$$


in (39) and using the notation $\boldsymbol{\alpha}_{i, j}=(i, j), \boldsymbol{\xi}(\boldsymbol{\omega})=\left(\xi_{x}\left(\omega_{x}\right), \xi_{v}\left(\omega_{v}\right)\right)=\left(k_{x}\left(\omega_{x}\right) \Delta x\right.$, $\left.k_{v}\left(\omega_{v}\right) \Delta v\right)$, we get

$$
\begin{aligned}
\frac{1}{|\Omega|^{1 / 2}} & \sum_{|\boldsymbol{\omega}| \leq \mathbf{N} / 2} \widehat{f}(\boldsymbol{\omega}) e^{i\left\langle\mathbf{k}(\boldsymbol{\omega}), \mathbf{z}_{i, j}\right\rangle} \\
= & \frac{1}{|\Omega|^{1 / 2}} \sum_{k, l} \sum_{|\boldsymbol{\omega}| \leq \mathbf{N} / 2} \widehat{\gamma}(\boldsymbol{\omega}) e^{i\left\langle\mathbf{k}(\boldsymbol{\omega}), \mathbf{z}_{k, l}\right\rangle} B_{m+1}(i-k) B_{m+1}(j-l) \\
= & \frac{1}{|\Omega|^{1 / 2}} \sum_{k, l} \sum_{|\boldsymbol{\omega}| \leq \mathbf{N} / 2} \widehat{\gamma}(\boldsymbol{\omega}) e^{i\left\langle\mathbf{k}(\boldsymbol{\omega}), \mathbf{z}_{i, j}\right\rangle} e^{-i\left\langle\boldsymbol{\xi}(\boldsymbol{\omega}), \boldsymbol{\alpha}_{i, j}-\boldsymbol{\alpha}_{k, l}\right\rangle} B_{m+1}(i-k) B_{m+1}(j-l) \\
= & \frac{1}{|\Omega|^{1 / 2}} \sum_{p, q} \sum_{|\boldsymbol{\omega}| \leq \mathbf{N} / 2} \widehat{\gamma}(\boldsymbol{\omega}) e^{i\left\langle\mathbf{k}(\boldsymbol{\omega}), \mathbf{z}_{i, j}\right\rangle} e^{-i\left\langle\boldsymbol{\xi}(\boldsymbol{\omega}), \boldsymbol{\alpha}_{p, q}\right\rangle} B_{m+1}(p) B^{m+1}(q),
\end{aligned}
$$

and it follows that

$$
\frac{1}{|\Omega|^{1 / 2}} \sum_{|\boldsymbol{\omega}| \leq \mathbf{N} / 2} \widehat{f}(\boldsymbol{\omega}) e^{i\left\langle\mathbf{k}(\boldsymbol{\omega}), \mathbf{z}_{i, j}\right\rangle}=\frac{1}{|\Omega|^{1 / 2}} \sum_{|\boldsymbol{\omega}| \leq \mathbf{N} / 2} \widehat{\gamma}(\boldsymbol{\omega}) e^{i\left\langle\mathbf{k}(\boldsymbol{\omega}), \mathbf{z}_{i, j}\right\rangle} \mathcal{D}(\boldsymbol{\omega}),
$$

where $\mathcal{D}(\boldsymbol{\omega})$ is defined by

$$
\mathcal{D}(\boldsymbol{\omega})=\sum_{p, q} e^{-i\left\langle\boldsymbol{\xi}(\boldsymbol{\omega}), \boldsymbol{\alpha}_{p, q}\right\rangle} B_{m+1}(p) B_{m+1}(q) .
$$

By multiplying (44) with its conjugate and $\Delta x \Delta v$, we get

$$
\begin{aligned}
& \frac{\Delta x \Delta v}{|\Omega|} \sum_{|\boldsymbol{\omega}| \leq \mathbf{N} / 2} \sum_{\left|\boldsymbol{\omega}^{\prime}\right| \leq \mathbf{N} / 2} \widehat{f}(\boldsymbol{\omega}) \overline{\widehat{f}\left(\boldsymbol{\omega}^{\prime}\right)} e^{i\left\langle\mathbf{k}(\boldsymbol{\omega})-\mathbf{k}\left(\boldsymbol{\omega}^{\prime}\right), \mathbf{z}_{i, j}\right\rangle} \\
& \quad=\frac{\Delta x \Delta v}{|\Omega|} \sum_{|\boldsymbol{\omega}| \leq \mathbf{N} / 2} \sum_{\left|\boldsymbol{\omega}^{\prime}\right| \leq \mathbf{N} / 2} e^{i\left\langle\mathbf{k}(\boldsymbol{\omega})-\mathbf{k}\left(\boldsymbol{\omega}^{\prime}\right), \mathbf{z}_{i, j}\right\rangle} \widehat{\gamma}(\boldsymbol{\omega}) \overline{\widehat{\gamma}\left(\boldsymbol{\omega}^{\prime}\right)} \mathcal{D}(\boldsymbol{\omega}) \overline{\mathcal{D}\left(\boldsymbol{\omega}^{\prime}\right)}
\end{aligned}
$$

As

$$
\frac{\Delta x \Delta v}{|\Omega|} \sum_{i=0}^{N_{x}} \sum_{j=0}^{N_{v}} e^{i\left\langle\mathbf{k}(\boldsymbol{\omega})-\mathbf{k}(\boldsymbol{\nu}), \mathbf{z}_{i, j}\right\rangle}=\delta_{\boldsymbol{\omega}, \boldsymbol{\nu}}
$$

we find the following relation which we will use later:

$$
\sum_{|\boldsymbol{\omega}| \leq \mathbf{N} / 2}|f(\boldsymbol{\omega})|^{2}=\sum_{|\boldsymbol{\omega}| \leq \mathbf{N} / 2}|\widehat{\gamma}(\boldsymbol{\omega})|^{2}|\mathcal{D}(\boldsymbol{\omega})|^{2} .
$$

We now want to compute $\left\|\mathscr{R}_{h} f\right\|_{L_{h}^{2}, \Delta_{h}^{\alpha, \mathbf{o}}}$ and $\left\|\mathscr{R}_{h} f\right\|_{L_{h}^{2}, \Delta_{h}^{\mathbf{0}, \boldsymbol{\beta}}}$. Beginning with $\left\|\mathscr{R}_{h} f\right\|_{L_{h}^{2}, \Delta_{h}^{\alpha, o}}$, we have

$$
\begin{aligned}
\left(\mathscr{R}_{h} f\right)_{i+\alpha_{j}, j}= & \sum_{k, l} \gamma_{k, l}(f) B_{m+1}\left(i+\alpha_{j}-k\right) B_{m+1}(j-l) \\
= & \frac{1}{|\Omega|^{1 / 2}} \sum_{k, l} \sum_{|\boldsymbol{\omega}| \leq \mathbf{N} / 2} \widehat{\gamma}(\boldsymbol{\omega}) e^{i\left\langle\mathbf{k}(\boldsymbol{\omega}), \mathbf{z}_{k, l}\right\rangle} B_{m+1}\left(i+\alpha_{j}-k\right) B_{m+1}(j-l) \\
= & \frac{1}{|\Omega|^{1 / 2}} \sum_{k, l} \sum_{|\boldsymbol{\omega}| \leq \mathbf{N} / 2} \widehat{\gamma}(\boldsymbol{\omega}) e^{i\left\langle\mathbf{k}(\boldsymbol{\omega}), \mathbf{z}_{i+\alpha_{j}, j}\right\rangle} e^{-i\left\langle\mathbf{k}(\boldsymbol{\omega}), \mathbf{z}_{i+\alpha_{j}-k, j-l}\right\rangle} \\
& \quad \times B_{m+1}\left(i+\alpha_{j}-k\right) B_{m+1}(j-l)
\end{aligned}
$$


so that

$$
\left(\mathscr{R}_{h} f\right)_{i+\alpha_{j}, j}=\frac{1}{|\Omega|^{1 / 2}} \sum_{|\boldsymbol{\omega}| \leq \mathbf{N} / 2} \widehat{\gamma}(\boldsymbol{\omega}) e^{i\left\langle\mathbf{k}(\boldsymbol{\omega}), \mathbf{z}_{i+\alpha_{j}, j}\right\rangle} \varrho\left(\omega_{x}, \alpha_{j}\right) D\left(\omega_{x}\right) D\left(\omega_{v}\right),
$$

where

$$
\varrho\left(\omega_{x}, \alpha_{j}\right)=\frac{\sum_{k} e^{i \xi_{x}\left(\omega_{x}\right)\left(i+\alpha_{j}-k\right)} B_{m+1}\left(i+\alpha_{j}-k\right)}{\sum_{p} e^{i \xi_{x}\left(\omega_{x}\right) p} B_{m+1}(p)}
$$

and

$$
D\left(\omega_{x}\right)=\sum_{p} e^{i \xi_{x}\left(\omega_{x}\right) p} B_{m+1}(p) .
$$

By multiplying (47) with its conjugate, summing on $i$ and $j$, and using (35) we get

$$
\begin{aligned}
& \left\|\mathscr{R}_{h} f\right\|_{L_{h}^{2}, \Delta_{h}^{\alpha, 0}}^{2} \\
& =\frac{\Delta v}{|2 R|} \sum_{\substack{|\boldsymbol{\omega}| \leq \mathbf{N} / 2 \\
\left|\omega_{v}^{\prime}\right| \leq N_{v} / 2 \\
j=0, \ldots, N_{v}}} \widehat{\gamma}(\boldsymbol{\omega}) \overline{\widehat{\gamma}\left(\omega_{x}, \omega_{v}^{\prime}\right)}\left|D\left(\omega_{x}\right)\right| D\left(\omega_{v}\right) \overline{D\left(\omega_{v}^{\prime}\right)}\left|\varrho\left(\alpha_{j}, \omega_{x}\right)\right|^{2} e^{i\left(k_{v}\left(\omega_{v}\right)-k_{v}\left(\omega_{v}^{\prime}\right)\right) v_{j}} .
\end{aligned}
$$

Using (36) and (46), the above becomes,

$$
\left\|\mathscr{R}_{h} f\right\|_{L_{h}^{2}, \Delta_{h}^{\boldsymbol{\alpha}}, \mathbf{o}}^{2} \leq \sum_{|\boldsymbol{\omega}| \leq \mathbf{N} / 2} \varrho_{\text {sup }}\left(\omega_{x}\right)|\widehat{\gamma}(\boldsymbol{\omega})|^{2}|\mathcal{D}(\boldsymbol{\omega})|^{2} \leq \varrho_{\text {sup }}\|f\|_{L_{h}^{2}(\Omega)}^{2}
$$

with

$$
\varrho_{\text {sup }}=\sup \left\{\left|\varrho\left(\alpha_{j}, \omega_{x}\right)\right|^{2}, 0 \leq \alpha_{j} \leq 1, \omega_{x} \in \mathbb{Z}\right\} .
$$

From Theorem [ 5 in Appendix B, we get $\left|\varrho_{\text {sup }}\right| \leq 1$ and consequently

$$
\left\|\mathscr{R}_{h} f\right\|_{L_{h}^{2}, \Delta_{h}^{\boldsymbol{\alpha}, 0}} \leq\|f\|_{L_{h}^{2}(\Omega)} \quad \text { and } \quad\left\|\mathscr{R}_{h} f\right\|_{L_{h}^{2}, \Delta_{h}^{\mathbf{0}, \boldsymbol{\beta}}} \leq\|f\|_{L_{h}^{2}(\Omega)} .
$$

5.2.2. Proof of the estimate (13). Let us now prove the estimate (13) for which we will use the Fourier analysis. First, we have

$$
c\|\gamma\|_{L_{h}^{2}(\Omega)} \leq\left\|\mathscr{R}_{h} f\right\|_{L^{2}(\Omega)} \leq C\|\gamma\|_{L_{h}^{2}(\Omega)} .
$$

Supposing again for convenience that $m+1$ is even, we get

$$
\begin{aligned}
& \int_{C_{i, j}}\left|\mathscr{R}_{h} f\right|^{2} d x d v \\
& \quad=\int_{C_{i, j}}\left|\sum_{|(k, \ell)| \leq(\mathbf{m}+\mathbf{1}) / 2} \gamma_{i+k, j+l} B_{m+1}(x / \Delta x-k) B_{m+1}(v / \Delta v-l)\right|^{2} d x d v .
\end{aligned}
$$

Since $B_{m+1}$ has its support on the interval $[-(m+1) / 2,(m+1) / 2]$, and by considering again a change of variables, we get

$$
\begin{aligned}
& \int_{C_{i, j}}\left|\mathscr{R}_{h} f\right|^{2} d x d v \\
& \quad=\Delta x \Delta v \int_{[-1 / 2,1 / 2]^{2}}\left|\sum_{|(k, \ell)| \leq(\mathbf{m}+\mathbf{1}) / 2} \gamma_{i+k, j+l} B_{m+1}(x-k) B_{m+1}(v-l)\right|^{2} d x d v .
\end{aligned}
$$


The right-hand side again defines the square of a norm, since we know that the ma$\operatorname{trix}\left[\sum_{k, l} B_{m+1}(i-k) B_{m+1}(j-l)\right]_{i, j=0}^{m+1 / 2, m+1 / 2}$ is invertible. Since all the quantities are now independent of $h$, we obtain the equivalence (48). It remains to prove that

$$
c_{1}\|\gamma\|_{L_{h}^{2}(\Omega)} \leq\|f\|_{L_{h}^{2}(\Omega)} \leq c_{2}\|\gamma\|_{L_{h}^{2}(\Omega)} .
$$

We will consider this relation in the discrete Fourier space, and will therefore establish that

$$
c_{1}\|\widehat{\gamma}\|_{L_{h}^{2}(\Omega)} \leq\|\widehat{f}\|_{L_{h}^{2}(\Omega)} \leq c_{2}\|\widehat{\gamma}\|_{L_{h}^{2}(\Omega)} .
$$

From the equality (46) and

$$
|\mathcal{D}(\boldsymbol{\omega})|=\left|\phi_{m+1}\left(0, \xi_{x}\left(\omega_{x}\right)\right)\right| \cdot\left|\phi_{m+1}\left(0, \xi_{v}\left(\omega_{v}\right)\right)\right|, \quad \phi_{m+1}(\alpha, \theta):=\sum_{p \in \mathbb{Z}} B_{m+1}(p+\alpha) e^{i \theta p}
$$

we see that the proof is completed, if we can establish that

$$
\inf _{\theta \in \mathbb{R}}\left|\phi_{m+1}(0, \theta)\right|>0 \text { and } \sup _{\theta \in \mathbb{R}}\left|\phi_{m+1}(0, \theta)\right|<\infty .
$$

The proof of this property for the B-splines is detailed at the end of the Appendix (Lemma 9).

\section{ApPendix A. LAGRANGe interpolation}

A.1. Theorems. Let $n \in \mathbb{N}^{*}, \theta \in \mathbb{R}$. We define $\varrho_{2 n}^{\theta} \in \mathbb{C}_{2 n}[X]$ such that $\varrho_{2 n}^{\theta}(j)=$ $\exp (i j \theta)$ for $j=-n, \ldots, n$, and $\varrho_{2 n-1}^{\theta} \in \mathbb{C}_{2 n-1}[X]$ such that $\varrho_{2 n-1}^{\theta}(j)=\exp (i j \theta)$ for $j=-n+1 / 2, \ldots, n-1 / 2$, and introduce $\xi:=1-\cos (\theta)$. We then have the following properties.

Theorem 3. We have

$$
\begin{aligned}
\left|\varrho_{2 n}^{\theta}(\zeta)\right|^{2}= & 1-\zeta^{2}\left(1-\zeta^{2}\right) \cdots\left(n^{2}-\zeta^{2}\right) \xi^{n+1} \\
& \times\left[c_{0}+c_{1} \xi\left(1-\zeta^{2}\right) c_{n-1} \xi^{n-1}\left(1-\zeta^{2}\right) \cdots\left((n-1)^{2}-\zeta^{2}\right)\right],
\end{aligned}
$$

with positive numbers $c_{i}, i=0, \ldots, n-1$, given by

$$
c_{i}=\frac{2^{n+i+1}}{(2 n) !(2 i+1) !(n+i+1)} \text {. }
$$

Theorem 4. We have

$$
\begin{aligned}
\left|\varrho_{2 n-1}^{\theta}(\zeta)\right|^{2}= & 1-\left(\left(\frac{1}{2}\right)^{2}-\zeta^{2}\right) \cdots\left(\left(\frac{2 n-1}{2}\right)^{2}-\zeta^{2}\right) \xi^{n} \\
& {\left[d_{0}+d_{1} \xi\left(\left(\frac{1}{2}\right)^{2}-\zeta^{2}\right)\right.} \\
& \left.+\cdots+d_{n-1} \xi^{n-1}\left(\left(\frac{1}{2}\right)^{2}-\zeta^{2}\right) \cdots\left(\left(\frac{2 n-3}{2}\right)^{2}-\zeta^{2}\right)\right]
\end{aligned}
$$

with positive numbers $d_{i}, i=0, \ldots n-1$, which are given by

$$
d_{i}=\frac{2^{n+i}}{(2 n-1) !(2 i) !(n+i)} .
$$


Corollary 1. We have

$$
\sup _{[-1 / 2,1 / 2]}\left|\varrho_{2 n-1}^{\theta}\right|=1, \quad \sup _{[-1,1]}\left|\varrho_{2 n}^{\theta}\right|=1 .
$$

Remark 3. Corollary 1 was already proven by Strang in [26]. The interest of these formulae is that it also says that the zone of stability is exactly $[-1,1]$ in the even case, and $[-1 / 2,1 / 2]$ in the odd case, at least for $n \leq 82$ (see the computer proof done in [19], where an analogous result is also given in the odd case). See also 14 for comments about the justification of the relation between the amplification factor (here $\varrho_{n}^{\theta}$ ) and the stability region.

Remark 4. The analysis which is carried out here for the Lagrange reconstruction on uniform grids does not apply if we take the same scheme on unstructured meshes (triangulation) by using high-order Lagrange interpolation on triangles. The determination of the stability region in that case remains an interesting open question. In the case of a constant advection, the semi-Lagrangian method can be interpreted as a finite difference method for which the stability and the convergence have been already studied in [26]. The analytical formula of the amplication factor indicates where the stability region is, and justifies the bad numerical behaviour when we use standard finite element reconstruction, whether it is on uniform grids or unstructured meshes. If the origin of the characteristic curves always falls in the region where the amplication factor is greater than one, the scheme will be unstable. Since in the semi-Lagrangian methods the characteristic origin is localised sometimes in a stable area and sometimes elsewhere, it is difficult to prove that there is convergence or not, but we observe that the solution becomes numerically unphysical. In this case the concept of convergence might have to be changed.

Here we present new proofs of Theorems 3 and 4 . We also give a very short proof of the Corollary 1 where the proof of the odd case is derived from the even case.

A.2. Proof. We first give an explicit formula for $\varrho_{2 n}^{\theta}$ which will be useful in the sequel. We recall that $\xi=1-\cos (\theta)$.

Lemma 6. Let $\varrho_{2 n}^{\theta} \in \mathbb{C}_{2 n}[X]$ such that $\varrho_{2 n}^{\theta}(j)=\exp (i j \theta)$ for $j=-n, \ldots, n$. We then have

$$
\varrho_{2 n}^{\theta}(z)=\varrho_{2 n-2}^{\theta}(z)+z\left(z^{2}-1\right) \cdots\left(z^{2}-(n-1)^{2}\right)(\varphi(z-n)+\bar{\varphi}(z+n)),
$$

with $\varphi=\frac{(-2)^{n-1}}{(2 n-1) !} \xi^{n-1}\left(e^{i \theta}-1\right)$.

Proof. We know that such a decomposition holds by expressing $\varrho_{2 n}^{\theta}$ in the basis: $1, z, z(z-1), z(z-1)(z+1) \ldots$, and since

$$
\overline{\varrho_{2 n}^{\theta}(-z)}=\varrho_{2 n}^{-\theta}(-z)=\varrho_{2 n}^{\theta}(z) .
$$

The coefficient $\varphi$ is given by the divided difference: $\varphi=\varrho_{2 n}^{\theta}[0,1, \ldots, n-1,-1, \ldots$, $n+1, n]$ which can also be expressed in terms of $\varrho_{2 n}^{\theta}(k)=\exp (i k \theta)$ :

$$
\varphi=\sum_{k=-n+1}^{n} \prod_{j=-n+1, j \neq k}^{n} \frac{1}{k-j} \exp (i k \theta) .
$$


Since $\prod_{j=-n+1}^{k-1}(k-j)=(n-1+k)$ ! and $\prod_{j=k+1}^{n}(k-j)=(-1)^{n-k}(n-k)$ !, we obtain that

$$
\varphi=\sum_{k=0}^{2 n-1} \frac{(-1)^{2 n-1-k}}{k !(2 n-1-k) !} \exp (i(-n+1+k) \theta)=\frac{(\exp (i \omega)-1)^{2 n-1}}{(2 n-1) !} \exp (i(-n+1) \theta)
$$

and

$$
(\exp (i \theta)-1)^{2 n-2} \exp (i(-n+1) \theta)=(\exp (i \theta / 2)-\exp (-i \theta / 2))^{2 n-2}=(-2)^{n-1} \xi^{n-1},
$$

where $\xi=2 \sin ^{2} \theta / 2$. We then have an the expression of $\varphi$.

In order to prove the algebraic formula (3), we first give a less precise form of it.

Proposition 1. We have the formula

$$
\begin{aligned}
\left|\varrho_{2 n}^{\theta}(x)\right|^{2}= & 1-x^{2}\left(1-x^{2}\right) \cdots\left(n^{2}-x^{2}\right) \\
& \times\left[C_{0}(\xi)+C_{1}(\xi)\left(1-x^{2}\right)+C_{n-1}(\xi)\left(1-x^{2}\right) \cdots\left((n-1)^{2}-x^{2}\right)\right],
\end{aligned}
$$

where the constants $C_{j}$ are polynomials in $\xi$ (for $j=0, \ldots, n-1$ ) and are given by

$$
C_{j}(\xi)=(-1)^{n+1+j} \sum_{k=1}^{j+1} \frac{R_{n}^{\prime}(k)}{\alpha_{k, j}}
$$

where the polynomial $R_{n}$ is defined by

$$
R_{n}\left(x^{2}\right):=\left|\varrho_{2 n}^{\theta}(x)\right|^{2}-1
$$

and $\alpha_{k, j}:=k^{2} \prod_{\ell=0 \ell \neq k}^{j+1}\left(k^{2}-\ell^{2}\right)^{2} \prod_{\ell=j+2}^{n}\left(k^{2}-\ell^{2}\right)$.

Proof. Since $\varrho_{2 n}^{\theta} \in \mathbb{C}_{2 n}[X]$ and since $\varrho_{2 n}^{\theta} \overline{\varrho_{2 n}^{\theta}}$ is even (from (50)), we deduce that $\varrho_{2 n}^{\theta} \overline{\varrho_{2 n}^{\theta}} \in \mathbb{C}_{2 n}\left[X^{2}\right]$. Now, we decompose $\varrho_{2 n}^{\theta}$ in the basis $x^{2}, x^{2}\left(x^{2}-1\right), \ldots, x^{2} \ldots$ $\left(x^{2}-n^{2}\right), x^{2} \cdots\left(x^{2}-n^{2}\right)\left(x^{2}-1\right), \ldots, x^{2} \cdots\left(x^{2}-n^{2}\right)\left(x^{2}-1\right) \cdots\left(x^{2}-(n-1)^{2}\right)$. We remark that the coefficients of $x^{2}, x^{2}\left(x^{2}-1\right), \ldots, x^{2} \cdots\left(x^{2}-(n-1)^{2}\right)$ are null, since

$$
\varrho_{2 n}^{\theta} \overline{\varrho_{2 n}^{\theta}}(j)-1=0, \quad j=0, \ldots, n
$$

In order to calculate the other coefficients, we fix $1>\varepsilon>0$ and we decompose $\varrho_{2 n}^{\theta}$ in the basis $x^{2}, x^{2}\left(x^{2}-1\right), \ldots, x^{2} \cdots\left(x^{2}-n^{2}\right), x^{2} \cdots\left(x^{2}-n^{2}\right)\left(x^{2}-1-\varepsilon\right), \ldots, x^{2} \cdots\left(x^{2}-\right.$ $\left.n^{2}\right)\left(x^{2}-1-\varepsilon\right) \cdots\left(x^{2}-(n-1)^{2}-\varepsilon\right)$. All the factors are now being distinct, and we can use the divided differences for evaluating the coefficients, and as $\varepsilon$ tends to 0 , we obtain

$$
(-1)^{n+1+j} C_{j}=\lim _{\varepsilon \rightarrow 0} \sum_{k=1}^{j+1} \frac{R_{n}(k+\varepsilon)}{\alpha_{k, j, \varepsilon}},
$$

with $\alpha_{k, j, \varepsilon}:=\prod_{\ell=0}^{n}\left(k^{2}+\varepsilon-\ell^{2}\right) \prod_{\ell=1 \ell \neq k}^{j+1}\left(k^{2}+\varepsilon-\ell^{2}-\varepsilon\right)$, since $R_{n}(j)=0$ for $j=0, \ldots, n$, and the formula (52) follows. It remains to prove that these coefficients can be expressed as polynomials in $\xi$. In fact, they are even in $\theta$, since $\varrho_{2 n}^{\theta} \overline{\varrho_{2 n}^{\theta}}$ is real and thus they are polynomials in $\cos \theta$ (the remaining term in $\sin \theta$ disappears with parity) and therefore in $\xi$.

In order to prove (3), we first prove:

Lemma 7. The $C_{j}$ are polynomials in $\xi$ of degree less than or equal to $n+1+j$. 
Proof. From (52), we know that each $C_{j}$ is a linear combination of

$$
R_{n}^{\prime}(1), \ldots, R_{n}^{\prime}\left((j+1)^{2}\right)
$$

where the coefficients are independent of $\theta$. Now, we have

$$
\varrho_{2 n}^{\theta}(x)-\exp (-i \theta) \varrho_{2 n}^{\theta}(x+1)=(\varphi+\bar{\varphi})(1-\exp (-i \theta)) w(x),
$$

where $w$ stands for

$$
w(x)=x(x+n) \prod_{k=1}^{n-1}\left(x^{2}-k^{2}\right)
$$

and $\varphi$ is defined in Lemma 6. Indeed, the member of the left vanishes at the points $-n,-n+1, \ldots, n-1$ and is of degree less than or equal to $2 n$. The leading coefficient of $\varrho_{2 n}^{\theta}$ is given by $\varphi+\bar{\varphi}$, from Lemma 6. Now, by differentiating the relation $R_{n}\left(x^{2}\right)=\varrho_{2 n}^{\theta}(x) \overline{\varrho_{2 n}^{\theta}(x)}-1$ and evaluating at an integer $k=0, \ldots, n-1$, we obtain

$$
2(k+1) R_{n}^{\prime}\left((k+1)^{2}\right)=e^{i(k+1) \theta} \overline{\varrho_{2 n}^{\theta \prime}(k+1)}+e^{-i(k+1) \theta} \varrho_{2 n}^{\theta \prime}(k+1) .
$$

Similar operations with (54) give:

$$
\varrho_{2 n}^{\theta \prime}(k+1)=\exp (i \theta) \varrho_{2 n}^{\theta \prime}(k)-(\exp (i \theta)-1)(\varphi+\bar{\varphi}) w^{\prime}(k) .
$$

We combine the two preceding equalities

$$
\begin{aligned}
& 2(k+1) R_{n}^{\prime}\left((k+1)^{2}\right)=2(\cos (k \theta)-\cos ((k+1) \theta))(\varphi+\bar{\varphi}) w^{\prime}(k) \\
& +e^{i k \theta} \overline{\varrho_{2 n}^{\theta \prime}(k)}+e^{-i k \theta} \varrho_{2 n}^{\theta \prime}(k)=2 k R_{n}^{\prime}\left(k^{2}\right)+c \xi^{n}\left(-\left(e^{-i \theta}+e^{i \theta}\right)^{k+1}+\sum_{\ell=-k}^{k} a_{\ell} e^{i \ell \theta}\right)
\end{aligned}
$$

with numbers $c, a_{-k}, \ldots, a_{k}$ independent of $\omega$, since $\varphi+\bar{\varphi}=\frac{(-2 \xi)^{n}}{(2 n-1) !}$. From the last expression, we see through induction that $R_{n}^{\prime}(k+1)$ is a polynomial of $\xi$ and cannot be of degree greater than $n+k+1$. That means, from (53), that the $C_{j}$ are polynomials of $\xi$ of degree less than $n+j+1$.

By using another decomposition, we obtain the converse.

Lemma 8. The coefficients $C_{j}$ are polynomials in $\xi$ of degree greater than or equal to $n+1+j$ (or are null).

Proof. We first prove by induction that $\left|\varrho_{2 n}^{\theta}\right|^{2}$ can be decomposed in sums and products of monomials of the form $\xi\left(z^{2}-k^{2}\right)$, where $k=0, \ldots, n$. Beginning with equation (49), we compute

$$
\left|\varrho_{2 n}^{\theta}(z)\right|^{2}=\left|\varrho_{2 n-2}^{\theta}(z)\right|^{2}+S_{n}(z)
$$

where $S_{n}(z)$ is a linear combination of the terms that are products of such monomials together with the products of

$$
z\left(e^{i \theta}-1\right)(z-k)\left(e^{-i \theta}-1\right)(z-\ell),
$$

where $k$ and $\ell$ are integers. Since $\left|e^{i \theta}-1\right|^{2}=\xi^{2}+\xi(2-\xi)=2 \xi$, the preceding term reads

$$
-2 \xi z^{2}(k+\ell) \text {. }
$$

The odd terms are simplified in $S_{n}$, as $\varrho_{2 n}^{\theta}$ is even, thus leading to the above decomposition. Now, a product of monomials of the form $\xi\left(z^{2}-k^{2}\right)$ can be expressed in the basis $1, z^{2}, z^{2}\left(z^{2}-1\right), \ldots, z^{2} \cdots\left(z^{2}-n^{2}\right), z^{2} \cdots\left(z^{2}-n^{2}\right)\left(z^{2}-1\right), \ldots, z^{2} \cdots\left(z^{2}-\right.$ 
$\left.n^{2}\right)\left(z^{2}-1\right) \cdots\left(z^{2}-(n-1)^{2}\right)$, and its coordinate along a vector $z^{2} \cdots\left(z^{2}-n^{2}\right)\left(z^{2}-\right.$ 1) $\cdots\left(z^{2}-k^{2}\right)$ is either null or of degree greater than or equal to $n+1+k$, if the product contains at least $n+1+k$ such monomials. We have just seen that $\left|\varrho_{2 n}^{\theta}\right|^{2}$ is a sum of products of such monomials. The lemma hence follows.

Now, from the two preceding lemmata, we have the existence of numbers $c_{j}$ such that $C_{j}=c_{j} \xi^{n+1+j}$, and we can therefore prove the Theorem 3 ,

Proof of Theorem 3. We are left with the determination of the numbers $c_{j}$. We obtain from (52) and (55)

$$
c_{j}=\frac{(-1)^{n+1+j} \Pi_{n+1+j} R_{n}^{\prime}\left((j+1)^{2}\right)}{a_{j, n}},
$$

where $\Pi_{n+1+j} R_{n}^{\prime}\left((j+1)^{2}\right)$ stands for the coordinates of $R_{n}^{\prime}\left((j+1)^{2}\right)$ over $\xi^{n+1+j}$, and

$$
\begin{aligned}
a_{j, n} & :=\prod_{k=0}^{j}\left((j+1)^{2}-k^{2}\right)^{2} \prod_{\ell=j+2}^{n}\left((j+1)^{2}-\ell^{2}\right) \\
& =\frac{(-1)^{n-j-1}(2 j+1) !(n-j-1) !(n+j+1) !}{2 j+2} .
\end{aligned}
$$

On the other hand, we obtain from (55)

$$
\Pi_{n+1+j} R_{n}^{\prime}\left((j+1)^{2}\right)=\frac{w^{\prime}(j) 2^{n+1+j}(-1)^{n+1+j}}{(2 n) ! 2(j+1)}
$$

where we compute $w^{\prime}(j)$ from

$$
w^{\prime}(j)=(-1)^{n-1-j}(j+n)(n-1-j) !(n-1+j) !
$$

By collecting the different terms together, we obtain the expression of $c_{j}$.

We can now also obtain Corollary 1 .

Proof of Corollary 1. The numbers $c_{i}$ in Theorem 3 are positive. We have, from Neville's algorithm,

$$
\varrho_{2 n+1}^{\theta}(\zeta)=\frac{(\zeta+n+1 / 2) \varrho_{2 n}^{\theta}(\zeta+1 / 2)-(\zeta-n-1 / 2) \varrho_{2 n}^{\theta}(\zeta-1 / 2)}{2 n+1} .
$$

Hence, if $\zeta \in[-1 / 2,1 / 2]$, we then obtain from the even case $\left|\varrho_{2 n+1}^{\theta}(\zeta)\right| \leq 1$.

We now give the proof of Theorem 4 .

Proof of Theorem 4. Following [26, first we will compute the derivative of the modulus of $\varrho_{2 n-1}^{\theta}$ by expressing it in a function of the real part $\mathcal{R}$ and the imaginary part $\mathcal{I}$ of $\varrho_{2 n-1}^{\theta}$. We compute precisely $\partial_{\theta}\left|\varrho_{2 n-1}^{\theta}\right|^{2}(\zeta)=2\left(\mathcal{I} \partial_{\theta} \mathcal{I}+\mathcal{R} \partial_{\theta} \mathcal{R}\right)(\zeta, \theta)$ where

$$
\begin{gathered}
\mathcal{R}(\zeta, \theta)=\sum_{k=-n+1 / 2}^{n-1 / 2} \ell_{k}(\zeta) \cos (k \theta), \quad \mathcal{I}(\zeta, \theta)=\sum_{k=-n+1 / 2}^{n-1 / 2} \ell_{k}(\zeta) \sin (k \theta), \\
\text { and } \ell_{k}(\zeta)=\prod_{\substack{i=-n+1 / 2 \\
i \neq k}}^{n-1 / 2} \frac{(\zeta-i)}{(k-i)}
\end{gathered}
$$


Let us now compute $\mathcal{R}, \mathcal{I}, \partial_{\theta} \mathcal{R}$ and $\partial_{\theta} \mathcal{I}$. Expressing the Lagrange interpolation polynomial in the Newton basis and using the notation $\varpi_{n-1 / 2}(\zeta)=$ $\left(\zeta^{2}-(1 / 2)^{2}\right) \cdots\left(\zeta^{2}-(n-1 / 2)^{2}\right)$, we get for $\zeta \in[0,1 / 2]$ and $\theta \in[0,2 \pi]$,

$$
\begin{aligned}
\mathcal{R}(\zeta, \theta)=\mathcal{R}_{-1 / 2} & +(\zeta+1 / 2) \Delta \mathcal{R}_{-1 / 2}+\frac{\varpi_{1 / 2}(\zeta)}{2 !} \Delta^{2} \mathcal{R}_{-3 / 2} \\
& +\cdots+\frac{\varpi_{n-3 / 2}(\zeta)(\zeta+n-1 / 2)}{(2 n-1) !} \Delta^{2 n-1} \mathcal{R}_{-n+1 / 2}
\end{aligned}
$$

and

$$
\begin{aligned}
\mathcal{I}(\zeta, \theta)=\mathcal{I}_{-1 / 2}+(\zeta+1 / 2) \Delta \mathcal{R}_{-1 / 2}+\frac{\varpi_{1 / 2}(\zeta)}{2 !} \Delta^{2} \mathcal{I}_{-3 / 2} \\
+\cdots+\frac{\varpi_{n-3 / 2}(\zeta)(\zeta+n-1 / 2)}{(2 n-1) !} \Delta^{2 n-1} \mathcal{I}_{-n+1 / 2}
\end{aligned}
$$

Let us show that $\Delta^{2 n-1} f_{-n+1 / 2}=0$ when $f(\zeta)$ is an even function:

$$
\begin{aligned}
\Delta^{2 n-1} f_{-n+1 / 2}= & \sum_{l=0}^{2 n-1}\left(\begin{array}{c}
2 n-1 \\
l
\end{array}\right)(-1)^{2 n-1-l} f_{l-n+1 / 2} \\
= & \sum_{l=0}^{n-1}\left(\begin{array}{c}
2 n-1 \\
l
\end{array}\right)(-1)^{2 n-1-l} f_{l-n+1 / 2} \\
& +\sum_{l=n}^{2 n-1}\left(\begin{array}{c}
2 n-1 \\
l
\end{array}\right)(-1)^{2 n-1-l} f_{l-n+1 / 2} \\
= & \sum_{l=0}^{n-1}\left(\begin{array}{c}
2 n-1 \\
l
\end{array}\right)(-1)^{2 n-1-l} f_{l-n+1 / 2} \\
& +\sum_{l=0}^{n-1}\left(\begin{array}{c}
2 n-1 \\
2 n-l-1
\end{array}\right)(-1)^{l} f_{n-l-1 / 2} \\
= & \sum_{l=0}^{n-1}\left(\begin{array}{c}
2 n-1 \\
l
\end{array}\right)(-1)^{l}\left(f_{k-1 / 2-l}-f_{l+1 / 2-k}\right)=0 .
\end{aligned}
$$

Let us find $\Delta^{2 k-2} \mathcal{R}_{-k+1 / 2}$ :

$$
\begin{aligned}
\Delta^{2 k-2} \mathcal{R}_{-k+1 / 2} & =\sum_{l=0}^{2 k-2}\left(\begin{array}{c}
2 k-2 \\
l
\end{array}\right)(-1)^{2 k-2-l} \mathcal{R}_{l-k+1 / 2} \\
& =\sum_{l=0}^{2 k-2}\left(\begin{array}{c}
2 k-2 \\
l
\end{array}\right)(-1)^{2 k-2-l} \sum_{j=-n+1 / 2}^{n-1 / 2} \ell_{j}(l-k+1 / 2) \cos (j \theta) \\
& =\Re e\left\{e^{i(-k+1 / 2) \theta} \sum_{l=0}^{2 k-2}\left(\begin{array}{c}
2 k-2 \\
l
\end{array}\right)(-1)^{2 k-2-l} e^{i l \theta}\right\} \\
& =(-1)^{k-1} \cos (\theta / 2) 2^{2 k-2}(\sin (\theta / 2))^{2 k-2} .
\end{aligned}
$$


We deduce that $\mathcal{R}(\zeta, \theta)$ can be rewritten as

$$
\mathcal{R}(\zeta, \theta)=\cos (\theta / 2)\left[1+\sum_{k=1}^{n-1} \frac{\varpi_{k-1 / 2}(\zeta)(-1)^{k} 2^{2 k} \sin ^{2 k}(\theta / 2)}{(2 k) !}\right] .
$$

Similar determination of $\Delta^{2 k-2} \mathcal{I}_{-k+1 / 2}$ and $\Delta^{2 k-1} \mathcal{I}_{-k+1 / 2}$ lead to the following expression for $\mathcal{I}$ :

$$
\begin{aligned}
\mathcal{I}(\zeta, \theta)= & -\sin (\theta / 2)+2(\zeta+1 / 2) \sin (\theta / 2) \\
& +\sum_{k=1}^{n-1} \frac{\varpi_{k-1 / 2}(\zeta) 2^{2 k}(-1)^{k+1} \sin ^{2 k+1}(\theta / 2)}{(2 k) !} \\
& +\sum_{k=1}^{n-1} \frac{\varpi_{k-1 / 2}(\zeta)(\zeta+k+1 / 2) 2^{2 k+1}(-1)^{k} \sin ^{2 k+1}(\theta / 2)}{(2 k+1) !} \\
= & \zeta \sum_{k=0}^{n-1} \frac{\varpi_{k-1 / 2}(\zeta) 2^{2 k+1}(-1)^{k} \sin ^{2 k+1}(\theta / 2)}{(2 k+1) !} .
\end{aligned}
$$

Differentiating $\mathcal{I}$ and $\mathcal{R}$ with respect to $\theta$ gives

$$
\begin{aligned}
\partial_{\theta} \mathcal{I}(\zeta, \theta)= & -\frac{1}{2} \cos (\theta / 2)+(\zeta+1 / 2) \cos (\theta / 2) \\
& +\sum_{k=1}^{n-1} \frac{\varpi_{k-1 / 2}(\zeta) 2^{2 k-1}(-1)^{k+1}(2 k+1) \sin ^{2 k}(\theta / 2) \cos (\theta / 2)}{(2 k) !} \\
& +\sum_{k=1}^{n-1} \frac{\varpi_{k-1 / 2}(\zeta)(\zeta+k+1 / 2) 2^{2 k}(-1)^{k}(2 k+1) \sin ^{2 k}(\theta / 2) \cos (\theta / 2)}{(2 k+1) !} \\
= & \zeta \cos \left(\frac{\theta}{2}\right)+\sum_{k=1}^{n-1} \frac{\varpi_{k-1 / 2}(\zeta) 2^{2 k}(-1)^{k} \sin ^{2 k}\left(\frac{\theta}{2}\right)}{(2 k+1) !} \\
& \times\left(-\frac{1}{2}(2 k+1) \cos \left(\frac{\theta}{2}\right)+\cos \left(\frac{\theta}{2}\right)\left(\zeta+k+\frac{1}{2}\right)\right) \\
= & \zeta \cos (\theta / 2)\left[1+\sum_{k=1}^{n-1} \frac{\varpi_{k-1 / 2}(\zeta) 2^{2 k}(-1)^{k} \sin ^{2 k}(\theta / 2)}{(2 k) !}\right]=\zeta \mathcal{R}
\end{aligned}
$$

and

$$
\begin{aligned}
\partial_{\theta} \mathcal{R}(\zeta, \theta)=- & \frac{\sin \left(\frac{\theta}{2}\right)}{2} \sum_{k=0}^{n-1} \frac{\varpi_{k-1 / 2}(\zeta) 2^{2 k}(-1)^{k} \sin ^{2 k}\left(\frac{\theta}{2}\right)}{(2 k) !} \\
& +\cos \left(\frac{\theta}{2}\right) \sum_{k=0}^{n-1} \frac{\varpi_{k-1 / 2}(\zeta) 2^{2 k}(-1)^{k} k \sin ^{2 k-1}\left(\frac{\theta}{2}\right) \cos \left(\frac{\theta}{2}\right)}{(2 k) !}
\end{aligned}
$$


Replacing $\cos ^{2}(\theta / 2)$ by $\left(1-\sin ^{2}(\theta / 2)\right)$, we rewrite the sum in $k$ from 0 to $n-2$ :

$$
\begin{aligned}
\partial_{\theta} \mathcal{R}(\zeta, \theta)= & \frac{\varpi_{n-3 / 2}(\zeta)(n-1 / 2)^{2} 2^{2 n-1}(-1)^{n} \sin ^{2 n-1}(\theta / 2)}{(2 n-1) !} \\
& +\sum_{k=0}^{n-2} \frac{\varpi_{k-1 / 2}(\zeta) 2^{2 k-1}(-1)^{k+1} \sin ^{2 k+1}(\theta / 2)}{(2 k) !} \\
& +\sum_{k=0}^{n-2} \frac{\varpi_{k-1 / 2}(\zeta) 2^{2 k}(-1)^{k+1} k \sin ^{2 k+1}(\theta / 2)}{(2 k) !} \\
& +\sum_{k=0}^{n-2} \frac{\varpi_{k-1 / 2}(\zeta) 2^{2 k+1}(-1)^{k+1} \sin ^{2 k+1}(\theta / 2)}{(2 k+1) !} .
\end{aligned}
$$

Reducing all the sums, we obtain

$$
\begin{aligned}
\partial_{\theta} \mathcal{R}(\zeta, \theta)= & -\frac{\varpi_{n-3 / 2}(\zeta)(n-1 / 2)^{2} 2^{2 n-1}(-1)^{n+1} \sin ^{2 n-1}(\theta / 2)}{(2 n-1) !} \\
& +\sum_{k=0}^{n-2} \frac{\varpi_{k-1 / 2}(\zeta) 2^{2 k}(-1)^{k} k \sin ^{2 k+1}(\theta / 2)}{(2 k) !} \\
& \times \overbrace{\left(-\left(k+\frac{1}{2}\right)-\frac{2\left(\zeta^{2}-\left(k+\frac{1}{2}\right)^{2}\right)}{2 k+1}\right)}^{-\frac{2 \zeta^{2}}{2 k+1}} \\
= & \frac{\varpi_{n-1 / 2}(\zeta) 2^{2 n-1}(-1)^{n+1} \sin ^{2 n-1}(\theta / 2)}{(2 n-1) !}-\zeta \mathcal{I} .
\end{aligned}
$$

Collecting the expression of $\mathcal{I}, \mathcal{R}, \partial_{\theta} \mathcal{I}$ and $\partial_{\theta} \mathcal{R}$ we have

$$
\begin{aligned}
\partial_{\theta}\left|\varrho_{2 n: 1}^{\theta}(\zeta)\right|^{2}= & -2^{2 n} \frac{(-1)^{n} \varpi_{n-1 / 2}(\zeta)}{(2 n-1) !} \sin ^{2 n-1}\left(\frac{\theta}{2}\right) \cos \\
& \times\left(\frac{\theta}{2}\right)\left[1+\sum_{k=1}^{n-1} \frac{\varpi_{k-1 / 2}(\zeta)(-1)^{k} 2^{2 k} \sin ^{2 k}(\theta / 2)}{(2 k) !}\right] .
\end{aligned}
$$

Integrating the above gives

$$
\begin{aligned}
\left|\varrho_{2 n-1}^{\theta}(\zeta)\right|^{2} & =g(\zeta)+-2^{2 n+1} \frac{(-1)^{n}}{2 n !} \varpi_{n-1 / 2}(\zeta) \sin ^{2 n}\left(\frac{\theta}{2}\right) \\
& -\frac{(-1)^{n} \varpi_{n-1 / 2}(\zeta)}{(2 n-1) !} \sum_{k=1}^{n-1} \frac{\varpi_{k-1 / 2}(\zeta)(-1)^{k} 2^{2(k+n)+1} \sin ^{2(k+l)}(\theta / 2)}{(2 k) ! 2(k+l)}
\end{aligned}
$$

As $\left|\varrho_{2 n-1}(\zeta, \theta=0)\right|^{2}=1$, we get $g(\zeta)=1$ and finally by setting $\xi=1-\cos \theta=$ $2 \sin ^{2}(\theta / 2)$, we find the desired result:

$$
\left|\varrho_{2 n-1}^{\theta}(\zeta)\right|^{2}=1-(-1)^{n} \varpi_{n-1 / 2}(\zeta) \xi^{n}\left(\sum_{k=0}^{n-1} \frac{2^{k+n+1}(-1)^{k} \xi^{k} \varpi_{k-1 / 2}(\zeta)}{(2 n-1) !(2 k) !(2(k+n))}\right) .
$$




\section{APPENDIX B. B-SPLINES INTERPOLATION}

We have used two important properties of the B-splines whose the proofs can be found in [21].

Theorem 5. Let $\theta \in \mathbb{R}$ and define $\Phi_{m}(\alpha)=\left|\sum_{k \in \mathbb{Z}} B_{m}(k+\alpha) e^{i k \theta}\right|^{2}$. Then, $\Phi_{m}$ admits its maximum on the integers.

Lemma 9. If we define $\phi(\alpha, \theta):=\left|\sum_{k \in \mathbb{Z}} B_{m+1}(k+\alpha) e^{i k \theta}\right|$, then we have

$$
0<\phi(0, \pi) \leq \phi(0, \theta)=\phi(0,0)=1, \quad \theta \in \mathbb{R} .
$$

\section{REFERENCES}

[1] B. J. C. Baxter, N. Sivakumar, On shifted cardinal interpolation by Gaussians and multiquadrics, J. Approx. Theory, 87 (1996), 36-59. MR1410611 (97e:41015)

[2] M. L. Begue, A. Ghizzo, P. Bertrand, Two-dimensional Vlasov simulation of Raman scattering and plasma beatwave acceleration on parallel computers, J. Comput. Phys., 151 (1999), 458-478.

[3] R. Bermejo, Analysis of an algorithm for the Galerkin-characteristic method, Numer. Math., 60 (1991), 163-194. MR1133578 (93e:65132)

[4] R. Bermejo, Analysis of a class of quasi-monotone and conservative semi-Lagrangian advection schemes, Numer. Math., 87 (2001), 597-623. MR1815727 (2001j:65130)

[5] N. Besse, Etude mathématique et numérique de l'équation de Vlasov non linéaire sur des maillages non structurés de l'espace des phases Ph.D. thesis of Institut de Recherche Mathématique Avancée, IRMA, Université Louis Pasteur, Strasbourg, 2003.

[6] N. Besse, Convergence of a semi-Lagrangian scheme for the one-dimensional Vlasov-Poisson system, SIAM, J. Numer. Anal., 42 (2004), 350-382. MR2051070(2005b:65098)

[7] N. Besse, Convergence of a high-order semi-Lagrangian scheme with propagation of gradients for the Vlasov-Poisson system, submitted.

[8] N. Besse, E. Sonnendrücker, Semi-Lagrangian schemes for the Vlasov equation on an unstructured mesh of phase space, J. Comput. Phys., 191 (2) (2003), 341-376. MR2016914 (2004j:82043)

[9] N. Besse, F. Filbet, M. Gutnic, I. Paun, E. Sonnendrücker, Adaptive numerical method for the Vlasov equation based on a multiresolution analysis, In F. Brezzi, A. Buffa, S. Escorsaro, and A. Murli editors, Numerical Mathematics and Advanced Applications ENUMATH 01, 437-446, Springer 2001.

[10] F. Bouchut, F. Golse, M. Pulvirenti, Kinetic equations and asymptotic theory, Series in Applied Mathematics, P.G. Ciarlet and P.-L. Lions (Eds.) Gauthier-Villars (2000). MR2065070 (2005d:82102)

[11] Carl de Boor, On the cardinal spline interpolant to $e^{i n t}$, SIAM J. Math. Anal, Vol. 7, No. 6. November 1976. MR0493056 (58:12096)

[12] M. Campos Pinto, M. Mehrenberger, Convergence of an adaptive scheme for the onedimensional Vlasov-Poisson system, submitted.

[13] C. Z. Cheng, G. Knorr, The integration of the Vlasov equation in configuration space, J. Comput. Phys., 22 (1976), 330-351.

[14] M. Falcone, R. Ferretti, Convergence analysis for a class of high-order semi-Lagrangian advection schemes, SIAM, J. Numer. Anal., 35 (1998), 909-940. MR1619910 (99c:65164)

[15] F. Filbet, E. Sonnendrücker, P. Bertrand, Conservative numerical schemes for the Vlasov equation, J. Comput. Phys., 172 (2001), 166-187. MR.1852326 (2002h:76106)

[16] E. Forest, J. Bengtsson, Application of the Yoshida-Ruth techniques to implicit integration and multi-map explicit integration, Phys. Lett. A 158 (1991), pp. 99-101. MR:1124345 (92f:70013)

[17] D. Goldman, T.J. Kaper, Nth-order operator splitting schemes and non reversible systems, SIAM J. Numer. Anal., 33 (1996), 349-367. MR1377257 (97a:65063)

[18] R. T. Glassey, The Cauchy problem in kinetic theory, Society for Industrial and Applied Mathematics (SIAM), Philadephia, PA, 1996. MR1379589(97i:82070)

[19] H. Hong, S. Steinberg, Accuracy and stability of polynomial interpolation schemes for advection equations, preprint, 2001. 
[20] P. G. Ciarlet, in Handbook of Numerical analysis, Finite element methods (part 1), Vol. II, P. G. Ciarlet and J. L. Lions (Eds.) North-Holland (1991). MR.1115235 (92f:65001)

[21] K. Jetter, S. D. Riemenschneider, N. Sivakumar, Schoenberg's exponential Euler spline curves, Proceedings of the Royal Society of Edinburgh, 118A, 21-33, 1991. MR.1113840 (92f:41021)

[22] F. J. Narcowich, N. Sivakumar, J. D. Ward, Stability results for scattered-data interpolation on euclidean spheres, Adv. Comput. Math., 8(3) (1998), 137-163. MR1628253(2000d:65016)

[23] I. J. Schoenberg, Cardinal interpolation and spline functions, Journal of Approximation Theory, 2, (1969), 167-206. MR0257616 (41:2266)

[24] E. Sonnendrücker, J. Roche, P. Bertrand, A. Ghizzo, The semi-Lagrangian method for the numerical resolution of the Vlasov equation. J. Comput. Phys. 149 (1999), no. 2, 201-220. MR.1672731 (99j:76100)

[25] A. Staniforth, J. Cote, Semi-Lagrangian integration schemes for atmospheric models-a review, Monthly Weather Review, 119 (1991), 2206-2223.

[26] G. Strang, Trigonometric polynomials and difference methods of maximum accuracy, J. Math. Phys, 41(2) (1962), 147-154.

[27] H. Yoshida, Construction of higher order sympletic integrators, Phys. Let. A 150 (1990), 262-268. MR.1078768 (91h:70014)

Institut de Recherche Mathematique Avancée, Université Louis Pasteur - CNRS, 7 rue René Descartes, 67084 Strasbourg Cedex, France

Current address: IECN UMR CNRS 7502 and LPMIA UMR CNRS 7040, Université Henri Poincaré Nancy I, Boulevard des Aiguillettes, B.P. 239 F-54506, Vandoeuvre-lès-Nancy, Cedex, France

E-mail address: besse@iecn.u-nancy.fr

Institut de Recherche Mathematique Avancée, Université Louis Pasteur - CNRS, 7 rue René Descartes, 67084 Strasbourg Cedex, France

E-mail address: mehrenbe@math.u-strasbg.fr 